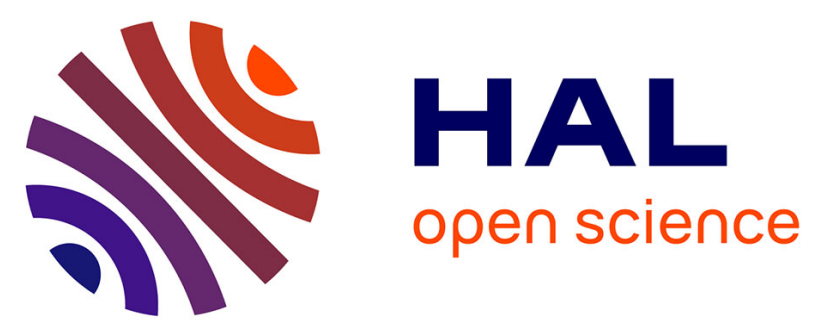

\title{
Seasonal variations in Titan's middle atmosphere during the northern spring derived from Cassini/CIRS observations
}

\author{
Sandrine Vinatier, Bruno Bézard, Sébastien Lebonnois, Nick A. Teanby, \\ Richard K. Achterberg, Nicolas Gorius, Andrei Mamoutkine, Ever Guandique, \\ Antoine Jolly, Donalds E. Jennings, et al.
}

\section{To cite this version:}

Sandrine Vinatier, Bruno Bézard, Sébastien Lebonnois, Nick A. Teanby, Richard K. Achterberg, et al.. Seasonal variations in Titan's middle atmosphere during the northern spring derived from Cassini/CIRS observations. Icarus, 2015, 250, pp.95-115. 10.1016/j.icarus.2014.11.019 . hal01102741

\section{HAL Id: hal-01102741 \\ https://hal.sorbonne-universite.fr/hal-01102741}

Submitted on 13 Jan 2015

HAL is a multi-disciplinary open access archive for the deposit and dissemination of scientific research documents, whether they are published or not. The documents may come from teaching and research institutions in France or abroad, or from public or private research centers.
L'archive ouverte pluridisciplinaire HAL, est destinée au dépôt et à la diffusion de documents scientifiques de niveau recherche, publiés ou non, émanant des établissements d'enseignement et de recherche français ou étrangers, des laboratoires publics ou privés. 


\title{
Seasonal variations in Titan's middle atmosphere during the northern spring derived from Cassini/CIRS observations
}

\author{
Sandrine Vinatier ${ }^{\mathrm{a}, *}$, Bruno Bézard ${ }^{\mathrm{a}}$, Sébastien Lebonnois ${ }^{\mathrm{b}}$, Nick Teanby ${ }^{\mathrm{c}}$, Richard \\ Achterberg $^{\mathrm{d}, \mathrm{e}}$, Nicolas Gorius ${ }^{\mathrm{e}}$, Andrei Mamoutkine ${ }^{\mathrm{e}}$, Ever Guandique ${ }^{\mathrm{e}}$, Antoine Jolly ${ }^{\mathrm{f}}$, \\ Don Jennings ${ }^{\mathrm{e}}$, Mike Flasar ${ }^{\mathrm{e}}$ \\ ${ }^{a}$ LESIA-Observatoire de Paris, CNRS, UPMC, Université Paris-Diderot, 5 place Jules Janssen, 92195 \\ Meudon, France. \\ ${ }^{b}$ Laboratoire de Météorologie Dynamique, Université Paris 06, France. \\ ${ }^{c}$ School of Earth Sciences, University of Bristol, Queen's Road, Bristol BS8 1RJ, UK. \\ ${ }^{d}$ Department of Astronomy, University of Maryland, College Park MD 20742, USA. \\ ${ }^{e}$ NASA/Goddard Space Flight Center, Code 693, Greenbelt, MD 20771, USA. \\ ${ }^{f}$ Laboratoire Interuniversitaire des Systmes Atmosphriques, UMR 7583 du CNRS, Universités Paris \\ Diderot et Paris-Est Créteil, Institut Pierre Simon Laplace, Créteil, France.
}

\begin{abstract}
We analyzed spectra acquired at the limb of Titan in the 2006 - 2013 period by the Cassini/Composite Infrared Spectrometer (CIRS) in order to monitor the seasonal evolution of the thermal, gas composition and aerosol spatial distributions. We are primarily interested here in the seasonal changes after the northern spring equinox and interpret our results in term of global circulation seasonal changes. Data cover the $600-1500 \mathrm{~cm}^{-1}$ spectral range at a resolution of 0.5 or $15.5 \mathrm{~cm}^{-1}$ and probe the $150-500 \mathrm{~km}$ vertical range with a vertical resolution of about $30 \mathrm{~km}$. Retrievals of the limb spectra acquired at $15.5 \mathrm{~cm}^{-1}$ resolution allowed us to derive eight global maps of temperature, aerosols and $\mathrm{C}_{2} \mathrm{H}_{2}, \mathrm{C}_{2} \mathrm{H}_{6}$ and HCN molecular mixing ratios between July 2009 and May 2013. In order to have a better understanding of the global changes taking place after the northern spring equinox, we analyzed $0.5 \mathrm{~cm}^{-1}$ resolution limb spectra to infer the mixing ratio profiles of 10 molecules for some latitudes. These profiles are compared with CIRS observations performed during the northern winter. Our observations are compatible with the coexistence of two circulation cells upwelling at mid-latitudes and downwelling at both poles from at last January 2010 to at least June 2010. One year later, in June 2011, there are indications that the global circulation had reversed compared to the winter situation, with a single pole-to-pole cell
\end{abstract}


upwelling at the north pole and downwelling at the south pole. Our observations show that in December 2011, this new pole-to-pole cell has settled with a downward velocity of 4.4 $\mathrm{mm} / \mathrm{s}$ at $450 \mathrm{~km}$ above the south pole. Therefore, in about two years after the equinox, the global circulation observed during the northern winter has totally reversed, which is in agreement with the predictions of general circulation models. We observe a sudden unexpected temperature decrease above the south pole in February 2012, which is probably related to the strong enhancement of molecular gas in this region, acting as radiative coolers. In July and November 2012, we observe a detached haze layer located around 320-330 km, which is comparable to the altitude of the detached haze layer observed by the Cassini Imaging Science Subsystem (ISS) in the UV.

Keywords: Titan, atmosphere, Infrared observations, Atmospheres, structure, Atmospheres, composition

Highlights: Two-cell dynamics exists in Titan's stratosphere from Jan. 2010 to June 2011. Reversal of the global dynamics occurs within 2 years after northern spring equinox. Timing of the dynamics changes agrees with General Circulation Model predictions. An unexpected cooling, starting in 2012, is observed at the south pole above $350 \mathrm{~km}$.

\section{Introduction}

Titan's atmosphere has one of the most complex chemistry in the solar system, which is based on the photodissociation of $\mathrm{N}_{2}$ (98\% in the middle atmosphere) and $\mathrm{CH}_{4}$ (between 1 and $1.5 \%$ in the middle atmosphere Niemann et al. (2010); Lellouch et al. (2014); Maltagliati et al. (2014); Bézard (2014)). In the upper atmosphere, typically above $800 \mathrm{~km}$, the combined photochemistry of $\mathrm{N}_{2}$ and $\mathrm{CH}_{4}$, through 80-200 nm solar radiation, energetic photoelectrons (produced by solar X-ray and EUV radiations) and Saturnian magnetospheric electrons, leads to the formation of the ionosphere, where very complex positive and negative ions (Waite et al., 2005; Coates et al., 2007) subsequently recombine to form complex

\footnotetext{
${ }^{*}$ Corresponding author

Email address: sandrine.vinatier@obspm.fr (Sandrine Vinatier)
} 
macromelecules that agglomerate into monomers and then in fractals aerosols while descending through the atmosphere (Lavvas et al., 2011). Dissociation of $\mathrm{N}_{2}$, leads to the formation of nitriles, $\mathrm{HCN}$ being by far the most abundant. Around $800 \mathrm{~km}, \mathrm{CH}_{4}$ photodissociation by the intense solar Lyman $\alpha$ line mostly drives the hydrocarbons photochemistry, while in the 200-300 km region, $\mathrm{CH}_{4}$ dissociation is driven by catalitic reactions via dissociation of $\mathrm{C}_{2} \mathrm{H}_{2}$ and other molecules whose radicals attack $\mathrm{CH}_{4}$. In the present study, we are interested in the region between 150 and $500 \mathrm{~km}$.

Dynamics, which is driven by the atmospheric thermal latitudinal gradients, redistributes molecules and aerosols, and also impacts the temperature field through vertical air motions and horizontal heat transport. Aerosols and molecules also affect the temperature by absorbing the solar radiation and emitting in the thermal infrared range. Therefore, complex couplings between photochemistry and dynamics exist in Titan's atmosphere. Associated with the meridional circulation and latitudinal thermal structure are strong zonal jets, particularly in the winter hemisphere (Flasar et al., 2005; Achterberg et al., 2008), which act as "a containment vessel" for the so-called polar vortex. Polar vortex confines molecular species, and because of the air subsidence occuring at the winter pole, these species are strongly enriched in the mesosphere/stratosphere inside the vortex. This was observed with CIRS during the winter above the north pole (Coustenis et al., 2007; Teanby et al., 2006, 2007, 2008b, 2009a; Vinatier et al., 2007a, 2010b).

Moreover, because of the $26.73^{\circ}$ obliquity of Titan, its atmosphere experiences strong seasonal variations of insolation. Titan's obliquity is comparable to the Earth's $23.5^{\circ}$ and in analogy to the dynamics of the Earth middle atmosphere, the 2D thermal and compositional structure has been interpreted in terms of pole-to-pole cell redistributing heat meridionally from the sunlit pole to the winter pole via adiabatic cooling and heating under solstitial conditions. During equinoxial conditions, a two cell pattern is predicted with upward motion and adiabatic cooling at mid-latitudes and downward motion with adiabatic heating at both poles. The transition from equinoxial to solstitial conditions is fairly rapid in the Earth middle atmosphere as the radiative time constant at the stratopause is only 5 days (or $\sim 0.015$ Earth year). In Titan's stratosphere, the radiative constant is $\sim 0.02$ Titan year over the 
altitude range 200-450 km (Strobel et al., 2010) and thus scales with Earth's time constant and validates the analogy. In agreement with this analogy, General Circulation Models (GCMs) of Titan's atmosphere (Hourdin et al., 2004; Lebonnois et al., 2012; Newman et al., 2011) predict that the total reversal of the pole-to-pole circulation occurs within about one or two years after the equinox.

The northern spring equinox occurred on 2009 August $11\left(\mathrm{Ls}=0^{\circ}\right)$. In June 2011, Teanby et al. (2012) observed for the first time above the south pole an enrichment at high altitude of $\mathrm{HC}_{3} \mathrm{~N}, \mathrm{HCN}$ and $\mathrm{C}_{2} \mathrm{H}_{2}$, which was the first evidence of the global post equinox dynamical changes with the beginning of an air subsidence above the south pole. Additionally, Jennings et al. (2012) detected with CIRS the emission feature at $220 \mathrm{~cm}^{-1}$ (attributed to a cloud of condensed material) at the south pole in July 2012, while it was only seen previously inside the northern polar vortex. In May 2012, a very interesting cloud, with an unexpected vertical extent in the stratosphere, was observed for the first time by the Cassini/Imaging Science Subsystem (ISS) above the south pole (West et al., 2013). This cloud is at least partly composed of HCN ice (de Kok et al., 2014).

Cassini also brought some information regarding the seasonal evolution of the aerosol spatial distribution around the equinox. During the northern winter, the meridional distribution of Titan's aerosols was also constrained from near infrared observations of the Cassini Visual and Infrared Mapping Spectrometer (VIMS) by Rannou et al. (2010) and from CIRS mid infrared observations (Vinatier et al., 2010a). From observations of ISS, West et al. (2011) inferred the seasonal evolution of the altitude of the detached haze layer which was localized around $500 \mathrm{~km}$ during the winter in 2007 and dropped to $380 \mathrm{~km}$ in 2010 .

One important question which this paper addresses is when does the transition from the single pole-to-pole circulation and breakdown of the winter polar vortex occur in Titan's stratosphere. We answer this question by deriving, from the analysis of CIRS limb spectra, the seasonal variations of temperature, molecular and aerosol mixing ratio spatial distributions between October 2006 (northern winter) and May 2013 (northern spring). Section 2 and 3 describe the observations used for this study and the retrieval method, respectively. Results and their implications regarding the global dynamic changes are detailed in Section 
4 and discussed in Section 5.

\section{Observations}

We analyze here the thermal emission of Titan's limb acquired by CIRS. CIRS is a Fourier Transform spectrometer that record spectra in the $20-1500 \mathrm{~cm}^{-1}$ spectral range, through three focal planes (FP1, FP3 and FP4). We focus here on the study of spectra acquired by the FP3 $\left(570-1125 \mathrm{~cm}^{-1}\right)$ and the FP4 $\left(1050-1495 \mathrm{~cm}^{-1}\right)$ focal planes, each composed of 10 adjacent detectors. During a limb observation, each detector array is positioned so that each detector probes a different altitude above a given latitude/longitude. At a given time on a limb observation, five detectors of FP3 and five detectors of FP4 acquire spectra simultaneously, the subsequent spectra are then acquired by the five other detectors of each focal planes. During about one to two hours, the 10 detectors arrays are positioned so that each detector record data from the same altitude. Then, FP3 and FP4 arrays are positioned so that they observe higher altitudes in the atmosphere. The field-of-view of each detector is $0.273 \mathrm{mrad}$. Limb observations used in this study, were acquired typically at distances between 100,000 and 180,000 km of Titan's surface, which results in a vertical resolution varying between 27 and $49 \mathrm{~km}$, which is comparable to the pressure scale height in Titan's stratosphere $(\sim 40 \mathrm{~km})$. During a single limb observation, lines-of-sight span altitudes between the surface and 600-700 km (depending of the spatial resolution of the observations). More details regarding CIRS and its different observing modes are given by Kunde et al. (1996); Flasar et al. (2004).

We utilized here two types of datasets: (i) spectra acquired at a spectral resolution of $0.5 \mathrm{~cm}^{-1}$ above a given latitude/longitude during a given flyby; (ii) spectra acquired at a spectral resolution of $15.5 \mathrm{~cm}^{-1}$, above several latitudes/longitudes during a given flyby. Both types of datasets are acquired during about 4 hours. It takes about 52 seconds to acquire a spectrum at a resolution of $0.5 \mathrm{~cm}^{-1}$, while a spectrum at a resolution of 15.5 $\mathrm{cm}^{-1}$ needs ten times less acquisition duration. As a result, during about 4 hours, a typical number of 20 latitudes are observed at a resolution of $15.5 \mathrm{~cm}^{-1}$, while only one latitude is probed with a spectral resolution of $0.5 \mathrm{~cm}^{-1}$. 
Both datasets have their own advantages. With observations at $0.5 \mathrm{~cm}^{-1}$ resolution, we are able to infer the mixing ratio profiles of about 10 molecules at a given surface coordinate and a given time, while spectra at $15.5 \mathrm{~cm}^{-1}$ resolution allows us to infer vertical mixing ratio profiles of about 3 molecules over an entire hemisphere with a typical spatial resolution of $5^{\circ}$ latitude at a given date.

In order to improve the signal-to-noise, we performed averages of limb spectra per detector, which corresponds to adjacent altitude bins of about $30-45 \mathrm{~km}$ (typically equal to the vertical resolution of the limb observations). Thus, about 10 to 15 averaged limb spectra were used to retrieved each vertical profiles of physical parameters.

Some of the observed limb spectra have a continuum with negative radiance in some spectral regions, mostly around $1000 \mathrm{~cm}^{-1}$, and other displayed continuum values that seemed to be shifted in intensity compared to other spectra. All spectra with unphysical or unusual spectral behaviors were removed from our averages.

\subsection{Observations at $0.5 \mathrm{~cm}^{-1}$}

In our previous study (Vinatier et al., 2010b), we used a dataset of limb spectra acquired at a spectral resolution of $0.5 \mathrm{~cm}^{-1}$ during the northern winter. In the present study, we extended this analysis to new selections of limb spectra acquired at comparable latitudes but later in the season. The objective is to infer the impact of the seasonal changes for 10 molecules $\left(\mathrm{C}_{2} \mathrm{H}_{2}, \mathrm{C}_{2} \mathrm{H}_{4}, \mathrm{C}_{2} \mathrm{H}_{6}, \mathrm{C}_{3} \mathrm{H}_{8}, \mathrm{CH}_{3} \mathrm{C}_{2} \mathrm{H}, \mathrm{C}_{4} \mathrm{H}_{2}, \mathrm{C}_{6} \mathrm{H}_{6}, \mathrm{HCN}, \mathrm{HC}_{3} \mathrm{~N}\right.$ and $\left.\mathrm{CO}_{2}\right)$ mixing ratio profiles in five different latitude regions: high northern latitudes (from $61^{\circ} \mathrm{N}$ to $88^{\circ} \mathrm{N}$ ), $46^{\circ} \mathrm{N}$, the Equator, $46^{\circ} \mathrm{S}$ and high southern latitudes (from 76 to $84^{\circ} \mathrm{S}$ ). Orbital solar longitudes of these observations vary from $\mathrm{L}_{s}=320^{\circ}$ (middle of the northern winter) to $\mathrm{L}_{s}=34^{\circ}$ (first third of the northern spring). Characteristic of these datasets are summarized in Table 1.

\subsection{Observations at $15.5 \mathrm{~cm}^{-1}$}

Our selections of limb spectra acquired at $15.5 \mathrm{~cm}^{-1}$ resolution are given in Table 2 . During Cassini's flybys with low inclination orbits (compared to the equatorial plane of 
Saturn), which occurred during the entire northern winter, the limb of an entire hemisphere was scanned. The second hemisphere was generally scanned during the following flyby. By combining both datasets acquired during consecutive or very close flybys, we were able to derive four global maps, from high southern to high northern latitudes in Dec. 2009 - Jan. 2010, June 2010, May - June 2011 and Jan. - Feb. 2012. We additionally used three other datasets acquired in July $2012\left(15^{\circ} \mathrm{S}-65^{\circ} \mathrm{N}\right)$, in November $2012\left(50^{\circ} \mathrm{S}-30^{\circ} \mathrm{N}\right)$ and in May $2013\left(25^{\circ} \mathrm{S}-20^{\circ} \mathrm{N}\right)$. After the beginning of 2012, limb observations did not probe the pole because of the the high inclined orbits of Cassini (which are designed to study the polar regions with nadir viewing). Polar regions will be probed with limb geometry anew in 2015, when Cassini orbits will go down in the equatorial plane of Saturn. The observations used here span the orbital solar longitudes from $\mathrm{L}_{s}=359^{\circ}$ (just before the northern spring equinox) to $45^{\circ}$ (in the middle of the northern spring). The latitude sampling that we applied to derive the maps was of $\sim 10^{\circ}$ at mid-latitude and about $\sim 5^{\circ}$ at high latitudes, in order to better probe the seasonal variations in the polar vortices.

\section{Retrieval method}

CIRS observes the thermal infrared emission of Titan's atmosphere, therefore, the intensity of molecular emission bands depends on both temperature and the mixing ratios of molecules responsible for emission bands. Continuum of CIRS spectra is due to both the collision-induced absorption of $\mathrm{N}_{2}-\mathrm{N}_{2}, \mathrm{~N}_{2}-\mathrm{CH}_{4}, \mathrm{CH}_{4}-\mathrm{CH}_{4}, \mathrm{~N}_{2}-\mathrm{H}_{2}$ and the opacity of aerosols. In order to constrain temperature, molecular mixing ratios and aerosol opacity, it is necessary to proceed in several steps. In a first step, we constrained simultaneously the temperature and the aerosol extinction vertical profiles. In a second step, we incorporated these profiles in our atmospheric model in order to derive the molecular mixing ratio profiles.

For both steps, we use inversion algorithms based on the fit of limb spectra with a lineby-line radiative transfer code. This radiative transfer code and the spectroscopic files used in the code are described in Vinatier et al. (2010b).

An improvement to the line data used in Vinatier et al. (2010a) is the inclusion of a new linelist for the $\nu_{6}+\nu_{8}$ combination band of $\mathrm{C}_{4} \mathrm{H}_{2}$ in the $1220-1260 \mathrm{~cm}^{-1}$ region. Inclusion 
of these bands allow a better fit to the methane $\mathrm{P}$ branch and more accurate temperature retrievals. We mostly observe this band at high latitude, where molecules are enriched by dynamics. Spectroscopic parameters of this combination band were provided by A. Jolly. The typical parallel band shape with two maxima at 1235 and $1247 \mathrm{~cm}^{-1}$ separated by a minimum at $1241 \mathrm{~cm}^{-1}$ is observed. The line list was derived from the work described in Jolly et al. (2010) on the $\mathrm{n}_{8}$ bending mode of diacetylene. Precise energy values were obtained through a global analysis of all levels including $\nu_{6}, \nu_{7}, \nu_{8}$ and $\nu_{9}$ enabling the calculation of positions and intensities for all lines belonging to the $\mathrm{n}_{6}+\mathrm{n}_{8}$ band. The intensity of the band was taken from the experimental determination by Khlifi et al. (1995) that was recently confirmed by Jolly et al. (2014).

\subsection{Temperature and aerosol extinction profiles retrievals}

We fitted the $\nu_{4} \mathrm{CH}_{4}$ band at $1305 \mathrm{~cm}^{-1}(7.7 \mu \mathrm{m})$ to constrain the temperature profile from several limb spectra acquired at different altitudes simultaneously and for a given latitude/longitude. A constant-with-height $\mathrm{CH}_{4}$ mixing ratio of $1.48 \%$ was measured in situ between 75 and $140 \mathrm{~km}$ by the Huygens Gas Chromatograph Mass Spectrometr (Niemann et al., 2010). In a recent study, Lellouch et al. (2014) used the far-infrared $\mathrm{CH}_{4}$ rotational lines observed in the CIRS FP1 focal plane to constrain the $\mathrm{CH}_{4}$ mixing ratio profiles at several latitudes. They inferred, during the northern winter, $\mathrm{CH}_{4}$ mixing ratios varying from $1 \%$ to $1.5 \%$ with a maximum at $\pm 30-35^{\circ}$ and polar latitudes and minimum at low latitudes and near $\pm 50-55^{\circ}$. They interpreted the local maxima at mid latitudes as the remanents from penetrative convective injection of methane clouds. No temporal variation of the $\mathrm{CH}_{4}$ mixing ratio could be inferred from their dataset (acquired during the northern winter) as they did not observed twice the same latitude. Another independent $\mathrm{CH}_{4}$ mixing ratio value was inferred by Maltagliati et al. (2014) from VIMS observations of solar occultations by Titan's atmosphere. They inferred a mean $\mathrm{CH}_{4}$ mixing ratio value of $1.25 \%$, which is lower than the in situ measurements but in agreement with the CIRS analysis of Lellouch et al. (2014). Very recently, Bézard (2014) inferred another $\mathrm{CH}_{4}$ mixing ratio of $1.44(+0.27 /-0.11) \%$ from the in situ measurements of the Huygens Descent Imager/Spectral Radiometer (DISR). 
In the present study, we chose to utilize the $\mathrm{CH}_{4}$ mixing ratio of $1.48 \%$, corresponding to the in situ GCMS measurements, as we did in our previous CIRS analysis.

The retrieval of the temperature and the aerosol optical depth vertical distribution are performed simultaneously by an inversion algorithm based on a constrained linear inversion method described in Conrath et al. (1998). We slightly modified our algorithm described in Vinatier et al. (2007a) in order to include the retrieval of the aerosol optical depth simultaneously with the temperature retrieval. The algorithm minimizes the quadratic differences $\left(\chi^{2}\right)$ between the observed and the calculated spectral intensity $(I)$, assuming initial temperature $(T)$ and aerosol optical depth $(\tau)$ profiles. From this minimization, two new profiles are derived for the $n^{\text {th }}$ iteration following the equations:

$$
\begin{aligned}
& T_{n}=T_{n-1}+\alpha S_{T} K^{T} C^{-1} \Delta I \\
& \tau_{n}=\tau_{n-1}+\beta S_{\tau} M^{T} C^{-1} \Delta I
\end{aligned}
$$

with:

$$
C=\alpha K S_{T} K^{T}+\beta M S_{\tau} M^{T}+E
$$

where $\mathrm{K}$ and $\mathrm{M}$ are the kernel matrices with $K_{i j}=\partial I_{i} / \partial T_{j}$ and $M_{i j}=\partial I_{i} / \partial \tau_{j}$ for the $i^{\text {th }}$ frequency and the $j^{\text {th }}$ level. Matrices $S_{T}$ and $S_{\tau}$ are Gaussian correlation matrices that provide a vertical filtering of the resulting temperature and aerosol extinction optical depth profiles, respectively. $E$ is a diagonal matrix with the diagonal elements equal to the square of the noise equivalent spectral radiance (NESR) of the CIRS spectrometer. $\alpha$ and $\beta$ are two scalar parameters that impose a constraint on $T_{n}$ and $\tau_{n}$ to lie more or less close to their respective initial guess, in order to obtain physically meaningful solutions, as the derivation of $T$ and $\tau$ profiles is a "ill-posed" problem. A typical number of 3 iterations generated a good convergence of the solution.

Ten to fifteen limb spectra per latitude/longitude coordinates were used to infer the thermal and the aerosol optical depth vertical profiles. Altitudes of the line-of-sight of these 
limb spectra were extracted from the CIRS database. Generally, these nominal altitudes did not allow us to infer a good fit of the P (1200-1280 $\left.\mathrm{cm}^{-1}\right)$ and Q (1280-1320 $\left.\mathrm{cm}^{-1}\right)$ branches of the $\nu_{4} \mathrm{CH}_{4}$ band simultaneously. We therefore had to apply an altitude shift on all the nominal altitudes of a given limb acquisition (i.e., the same for all the 10 detectors of the FP4 array) in order to fit properly the observed limb spectra (see Vinatier et al. (2007a) for more details). This shift has two origins: the CIRS navigation pointing error and the calculated pressure/altitude grid from hydrostatic equilibrium based on our a priori thermal profile outside the regions probed by CIRS, which is equal to the in situ temperature profile measured by the Huygens Atmospheric Structure Instrument (HASI) instrument (Fulchignoni et al., 2005) outside regions probed by CIRS. Some example of the shift values that were applied are given in Table 1 of Vinatier et al. (2010b). In the present work, we generally applied shifts with amplitudes lower than $20 \mathrm{~km}$ (with a predominance of values lower than $10 \mathrm{~km}$ ), except for limb spectra acquired during the T80 flyby, for which we had to apply shifts comprised between -60 and $-130 \mathrm{~km}$, which suggests a navigation pointing error in that case.

Temperature and aerosol optical depth profiles were derived from the simultaneous fits of the 1080-1120 and 1200-1330 $\mathrm{cm}^{-1}$ spectral range. Aerosol opacity is mostly constrained by the fit of the 1080-1120 $\mathrm{cm}^{-1}$ spectral range, a region mostly free of gas emission bands. While temperature is mostly constrained from the fit of the $1200-1330 \mathrm{~cm}^{-1}$ spectral range, which displays the $\mathrm{P}, \mathrm{Q}$ and part of the $\mathrm{R}$ branches of the $\nu_{4} \mathrm{CH}_{4}$ band. Impact of the aerosol opacity on the continuum intensity can be visualized in Fig. 1 of Vinatier et al. (2010a).

We utilized the spectral dependance of the aerosol extinction coefficient displayed in Vinatier et al. (2012) (Fig. 1b) for all the limb spectra of our dataset acquired at low and mid latitudes. At very high latitude, typically higher than $70 \mathrm{~N}$, we performed a very similar study to the one done by Vinatier et al. (2010a) to infer modified spectral dependences of the aerosol extinction, which allowed us to get a better fit of the limb data (both in the case of 0.5 and $15.5 \mathrm{~cm}^{-1}$ resolution).

The retrieved temperature and aerosol optical depth profiles were then incorporated in 
our atmospheric model in order to fit the spectral emission bands of molecular gases in order to constrain their mixing ratios profiles. We applied the altitude shifts that were constrained from the fit of the $\nu_{4} \mathrm{CH}_{4}$ band to the nominal altitudes of the FP3 spectra acquired simultaneously with the FP4 spectra.

\subsection{Molecular gas mixing ratio profiles retrievals}

For limb spectra acquired at $0.5 \mathrm{~cm}^{-1}$ resolution, it is possible to infer the mixing ratio profiles of $\mathrm{C}_{2} \mathrm{H}_{2}, \mathrm{C}_{2} \mathrm{H}_{4}, \mathrm{C}_{2} \mathrm{H}_{6}, \mathrm{C}_{3} \mathrm{H}_{8}, \mathrm{CH}_{3} \mathrm{C}_{2} \mathrm{H}, \mathrm{C}_{4} \mathrm{H}_{2}, \mathrm{C}_{6} \mathrm{H}_{6}, \mathrm{HCN}, \mathrm{HC}_{3} \mathrm{~N}$ and $\mathrm{CO}_{2}$ as the spectral resolution is high enough to separate unambiguously the emission bands of all these molecular gases (see Fig. 1, upper panel).

Limb spectra acquired at $15.5 \mathrm{~cm}^{-1}$ resolution clearly show the spectral signatures of the same molecules, but some of the emission bands are mixed, as it is the case for $\mathrm{CH}_{3} \mathrm{C}_{2} \mathrm{H}$ and $\mathrm{C}_{4} \mathrm{H}_{2}$, or $\mathrm{HC}_{3} \mathrm{~N}, \mathrm{CO}_{2}$ and $\mathrm{C}_{6} \mathrm{H}_{6}$ (see Fig. 1, lower panel). For the analysis of these low spectral resolution data, we therefore limited the retrieval to the $\mathrm{C}_{2} \mathrm{H}_{2}, \mathrm{C}_{2} \mathrm{H}_{6}$ and $\mathrm{HCN}$ molecular mixing ratios.

Regarding the inversion of the $0.5 \mathrm{~cm}^{-1}$ resolution spectra, we used a retrieval method similar to the one described by Vinatier et al. (2007a, 2010b). Our atmospheric model incorporated the temperature and haze optical depth profiles retrieved in the first step of our analysis (Section 3.1). Temperature profile was set as a fixed parameter, while the aerosol optical depth profile retrieved from the FP4 was used as an initial guess. We used several spectral ranges including a few molecular bands each (see Vinatier et al. (2010b) for the exact values of these ranges) to retrieve up to three profiles (the aerosol optical depth profile and one or two molecular mixing ratio profiles) simultaneously.

For the inversion of the $15.5 \mathrm{~cm}^{-1}$ resolution spectra, we retrieved simultaneously the aerosol optical depth, the $\mathrm{C}_{2} \mathrm{H}_{2}$ and $\mathrm{C}_{2} \mathrm{H}_{6}$ mixing ratio profiles from the $700-750 \mathrm{~cm}^{-1}$ and $780-850 \mathrm{~cm}^{-1}$ spectral ranges simultaneously. The $750-780 \mathrm{~cm}^{-1}$ spectral region was removed from this inversion in order to avoid the $765 \mathrm{~cm}^{-1}$ noise spike (see Fig. 1 upper panel). The 700-750 $\mathrm{cm}^{-1}$ spectral range is mostly sensitive to the $\mathrm{C}_{2} \mathrm{H}_{2}$ and $\mathrm{HCN}$ mixing ratio, while the $780-850 \mathrm{~cm}^{-1}$ range is sensitive to the $\mathrm{C}_{2} \mathrm{H}_{6}$ mixing ratio value (see Fig. 1). Because 
of the low spectral resolution, the constraint on the aerosol optical depth is poorer than for a resolution of $0.5 \mathrm{~cm}^{-1}$ because it is quite difficult to separate the aerosols contribution to the continuum from the molecular band contributions. In our first tests, we decided not to retrieve the aerosol optical depth profiles and to use the one retrieved from the FP4 spectral range as a fixed parameter. But in some cases, we noticed that the fit of the $750-850 \mathrm{~cm}^{-1}$ region was inadequate. We therefore decided to also retrieve, simultaneously with the $\mathrm{C}_{2} \mathrm{H}_{2}$ and $\mathrm{C}_{2} \mathrm{H}_{6}$ mixing ratio profiles, the aerosol optical depth profile from the $700-750 \mathrm{~cm}^{-1}$ and $780-850 \mathrm{~cm}^{-1}$ spectral ranges, while constraining the retrieved profile to lie close to the initial guess. In this way, we obtained better fits of the observed spectra, while the retrieved aerosol optical depth profiles were generally slightly shifted on the entire altitude range compared to the aerosol initial guess. After having derived the aerosol optical depth and the $\mathrm{C}_{2} \mathrm{H}_{2}$ and $\mathrm{C}_{2} \mathrm{H}_{6}$ mixing ratio profiles, we incorporated them in the atmospheric model as fixed parameters and we performed a retrieval of the HCN mixing ratio profile from the 700-725 $\mathrm{cm}^{-1}$ spectral range. In a new iteration, we then performed a new retrieval of the aerosol optical depth and the $\mathrm{C}_{2} \mathrm{H}_{2}$ and $\mathrm{C}_{2} \mathrm{H}_{6}$ mixing ratio profiles with the atmospheric model including the retrieved $\mathrm{HCN}$ profile.

\section{Results}

\subsection{Results from the $15.5 \mathrm{~cm}^{-1}$ spectra: global maps}

\subsubsection{Temperature maps}

Fig. 2 displays the resulting 2D-maps (latitude/pressure) of temperature between northern winter and spring. The top left map, which corresponds to the northern winter season, is adapted from Vinatier et al. (2010b). It was inferred from several limb datasets acquired at a $0.5 \mathrm{~cm}^{-1}$ spectral resolution at different dates (from February 2005 to May 2008). Other maps are derived from the present analysis of $15.5 \mathrm{~cm}^{-1}$ resolution limb spectra. The latitudinal sampling of each map is given by the red marks. Error bars on the top left map do not exceed $1 \mathrm{~K}$ and those of other maps are lower than $2 \mathrm{~K}$.

A first noteworthy feature is the seasonal evolution of the polar temperatures. During the northern winter, a very warm stratopause was observed at 0.01 mbar $(400 \mathrm{~km})$ with a 
maximum temperature above the north pole of 206 K. Such a high temperature resulted from an adiabatic heating due to the descending branch of the pole-to-pole circulation cell (Flasar and Conrath, 1990; Flasar et al., 2005; Achterberg et al., 2008, 2011). The temperature of this 0.01-mbar warm region decreased with time from winter to June 2010 (see Achterberg et al. (2011) for the seasonal variations between July 2004 and December 2009), when it became comparable to the mid-latitude temperature. This north pole temperature decrease was interpreted by Achterberg et al. (2011) by a weakening of the subsidence within the descending branch of the middle atmospheric meridional circulation. At the south pole, during winter and up to March 2008, a well-defined stratopause was observed around 0.1 mbar with a temperature of $\sim 185 \mathrm{~K}$ (see Achterberg et al. (2011), Fig. 5). Unfortunately, the south pole was not observed in limb geometry viewing by CIRS between March 2008 and January 2010. In January 2010, we derive a different stratopause pattern with two local temperature maxima above $80^{\circ} \mathrm{S}$ at 0.2 and 0.005 mbar both with temperatures of 175 $\mathrm{K}$ each. We believe that the high altitude local temperature maximum (at 0.005 mbar) is due to an adiabatic heating caused by a descending branch there. As southern autumn was progressing after June 2010, the $0.2 \mathrm{mbar}$ local temperature maximum disappeared and the 0.005 mbar one was observed deeper, at 0.01 mbar with temperature progressively reinforced by $6 \mathrm{~K}$ up to June 2011. This temperature increase above the south pole observed since January 2010 suggests that the descending branch of the meridional circulation at the south pole appeared before January 2010, five months (or $\sim 9$ Titan's days) after the southern autumn equinox. Actually, in January 2010, as the 2-D composition maps are consistent with a descending branch at the north pole (see Section 4.1.2), we think that two circulation cells subsiding at both poles were occuring in Titan's middle atmosphere at this date and probably up to a least June 2010. After the establishment of the descending branch above the south pole, one would expect warmer temperatures later in the southern autumn season, because of the adiabatic heating being more and more efficient. This temperature increase was observed up to June 2011, but suddenly, in February 2012, this warm zone unexpectidly disappeared, and a temperature of $157 \mathrm{~K}$ (i.e. $26 \mathrm{~K}$ colder than in June 2011) was observed at 0.01 mbar at $82^{\circ} \mathrm{S}$. As from General Circulation Models, the air subsidence above the 
south pole should maintain up to the southern spring equinox in 2025, we suggest that this cooling results from a strong radiative cooling (exceeding the adiabatic heating), due to the simultaneous high enhancement of radiative coolers in the same region (see Section 4.1.2). Unfortunately, because of the high inclination of the Cassini orbits relative to Saturn's ring plane, the south pole has not been observed after this date with limb geometry viewing. Limb viewing of the poles will resume in 2015, when Cassini's orbits descend to the Titan orbital plane.

At low and mid-latitudes, we also observe a seasonal evolution of the stratopause (the local maximum temperature) both in temperature and location. During the northern winter, stratopause had a maximum temperature of $\sim 185 \mathrm{~K}$ and was localized around 0.07 mbar $(\sim 320 \mathrm{~km})$. After the northern spring equinox, the maps in Dec. 2009 - Jan. 2010 and June 2010 show a stratopause temperature decrease of $\sim 5 \mathrm{~K}$ and a deeper location at 0.2 mbar (altitude $\sim 270 \mathrm{~km}$ ). This lowering of the stratopause altitude was first observed by Achterberg et al. (2011) a few months before equinox in May 2009 (see their fig. 4). In May-June 2011 and Jan.-Feb. 2012, the stratopause altitude continued to decrease towards the 0.3 mbar level $(\sim 240 \mathrm{~km})$ with a temperature of $175 \mathrm{~K}$ at mid southern latitudes and $180 \mathrm{~K}$ near the equator. Later, in July and November 2012, the equatorial stratopause altitude continued to decrease and was observed at 0.4 mbar $(\sim 225 \mathrm{~km})$ with a maximum temperature of $179 \mathrm{~K}$. Additionally, a secondary temperature maximum was also observed at 0.04 mbar $(\sim 350 \mathrm{~km})$ reaching $176 \mathrm{~K}$, which could be related to the radiative heating of a detached haze layer in this region (see Section 4.1.3 and 5.2). In May 2013, a similar temperature pattern was observed. Thus, in summary, from the northern winter to the mid-spring, the mid-latitude stratopause temperature decreased from 185 to $177 \mathrm{~K}$ and the stratopause descended from 0.06 to $\sim 0.5$ mbar $(320$ to $215 \mathrm{~km})$. Such a global temperature decrease could be attributed to the reduction of about $13 \%$ of the solar flux at Titan between October 2006 and May 2013, because of Saturn's orbital eccentricity.

In the lower stratosphere, below the 1-mbar level, the temperature seasonal evolution is also well marked. At high northern latitudes, during the northern winter, we observed temperatures as cold as $145 \mathrm{~K}$ at 1 mbar. Later, as the northern spring progressed, the 
deeper stratosphere continuously warmed up and reached temperature of $157 \mathrm{~K}$ in February 2012 at the same pressure level. During the same period, while southern autumn progressed, deep stratospheric temperatures at the south pole decreased from $\sim 170 \mathrm{~K}$ at 1 mbar in 2008 (Achterberg et al., 2011) to $150 \mathrm{~K}$ in February 2012, being then almost comparable to the observed temperature at the north pole during the northern winter. Actually, south polar temperature continued to decrease later to reach about $130 \mathrm{~K}$ at 1 mbar in October 2013 (de Kok et al., 2014), which is colder than the north pole temperature observed during the northern winter.

\subsubsection{Molecular gas mixing ratio maps: $\mathrm{C}_{2} \mathrm{H}_{2}, \mathrm{C}_{2} \mathrm{H}_{6}$ and $\mathrm{HCN}$}

Fig. 3, 4, and 5 display the pressure/latitudes maps of $\mathrm{C}_{2} \mathrm{H}_{2}, \mathrm{HCN}$ and $\mathrm{C}_{2} \mathrm{H}_{6}$ mixing ratios, respectively. The top left plots of these figures are adapted from the results of Vinatier et al. (2010b) and represent the spatial distributions of the molecular mixing ratios during the northern winter, which were derived from $0.5 \mathrm{~cm}^{-1}$ resolution limb spectra acquired between February 2005 and May 2008. $\mathrm{C}_{2} \mathrm{H}_{2}$, $\mathrm{HCN}$ and $\mathrm{C}_{2} \mathrm{H}_{6}$ have chemical lifetimes at 300 km ( 0.1 mbar) equal to 10, 13 and 54 years, respectively (Wilson and Atreya, 2004). These lifetimes are much larger (at least 6 times) than the meridional overturning timescale, which is typically a few Titan's days at 1 mbar (Newman et al., 2011; Lebonnois et al., 2012). Therefore, the global spatial distribution of these molecules in the middle stratosphere is mostly affected by the global dynamics and can be considered as good tracers of the global dynamical changes.

Because of strong zonal winds, the northern polar vortex was quite confined above the north pole during the winter and the descending branch occurring there, bringing enriched air from higher altitudes were molecules are formed to the deeper levels, was responsible for an enhancement of all molecular gases in this region. In December 2009, after the northern spring equinox, more pronounced polar enrichments were observed for $\mathrm{C}_{2} \mathrm{H}_{2}, \mathrm{HCN}$ and $\mathrm{C}_{2} \mathrm{H}_{6}$, and also for $\mathrm{C}_{2} \mathrm{H}_{4}, \mathrm{CH}_{3} \mathrm{C}_{2} \mathrm{H}, \mathrm{C}_{4} \mathrm{H}_{2}, \mathrm{HC}_{3} \mathrm{~N}$ and $\mathrm{C}_{6} \mathrm{H}_{6}$ (see Section 4.2.1). This enriched zone was then less confined around the north pole compared to the winter observations, which is in agreement with the predicted decrease of the strength of the north zonal winds 
around equinox (Newman et al., 2011; Lebonnois et al., 2012) then reducing the polar vortex confinment efficiency. Later, in June 2010, while northern spring was progressing, this north pole enriched zone moved deeper, suggesting that the air was still subsiding there. This result combined with the observed adiabatic heating at the south pole at the same date (see Section 4.1.1) suggest a two-cell pattern with subsidence at both poles. The location of the upwelling branches can not be inferred from our data.

At very high southern latitudes (higher than $70^{\circ} \mathrm{S}$ ), an enhancement of molecular gas is observed in June 2011 for $\mathrm{HCN}$ and $\mathrm{C}_{2} \mathrm{H}_{2}$ above the 0.004 mbar level $(430 \mathrm{~km})$. This enriched zone extended down to the 0.03 mbar level in January 2012, where $\mathrm{C}_{2} \mathrm{H}_{6}$ was also observed to be enriched. This is explained by the air subsidence occuring at the south pole since January 2010 (see Section 4.1.1), which transports the high altitude enriched air downward. Later, in November 2012, a strong enhancement of $\mathrm{C}_{2} \mathrm{H}_{2}, \mathrm{C}_{2} \mathrm{H}_{6}$ and $\mathrm{HCN}$ is observed from $40^{\circ} \mathrm{S}$ to $50^{\circ} \mathrm{S}$ above the $0.01 \mathrm{mbar}$ level $(400 \mathrm{~km}$ ), and seems to have extended toward $25^{\circ} \mathrm{S}$ in May 2013. This suggests that the enriched zone observed above the south pole in June 2011 and February 2012 extended towards mid-southern latitudes while autumn progressed. From this step of our interpretation of the seasonal evolution of thermal and molecular mixing ratio maps, we know that the southern descending branch was observed since January 2010 (because of the adiabatic heating observed around 0.005 mbar) and the northern descending branch was probably still being maintained in June 2010 (because of the molecular enhancement observed deeper at high northern latitude compared to January 2010). But in June 2011, we observe an enrichment of $\mathrm{C}_{2} \mathrm{H}_{2}$ and $\mathrm{HCN}$ at high altitude and mid northern latitude, which suggests that the enriched air of the north pole was transported towards the equator in the 0.05-0.001 mbar region (with a stronger impact on $\mathrm{HCN}$ ) by a single circulation cell upwelling at the north pole. This enrichment accentuated later as expected by the strengthening of the dynamics after the settlement of the single circulation cell regime. We therefore believe that the global dynamics has reversed before June 2011, less than two years (or about $~ 41$ Titan's days) after the northern spring equinox. Surprisingly, in July 2012, a depletion of the three molecules is observed above the $0.01 \mathrm{mbar}$ level in the northern hemisphere. A data problem was ruled out because similar results were 
retrieved from limb spectra acquired at $0.5 \mathrm{~cm}^{-1}$ spectral resolution (see Section 4.2 .2 for the observation at $\left.45^{\circ} \mathrm{N}\right)$.

\subsubsection{Aerosol extinction and mass mixing ratio maps}

Figures 6 and 7 present the extinction coefficient $\left(\right.$ in $\mathrm{cm}^{-1}$ ) at $1090 \mathrm{~cm}^{-1}$ and the mass mixing ratio of aerosols, respectively. Aerosol extinction is derived from the continuum of the CIRS limb spectra in the $1080-1120 \mathrm{~cm}^{-1}$ spectral region, simultaneously with the temperature profile retrieval, as described in Section 3.1. Results regarding the aerosol extinction above the 0.01 mbar level must be regarded with caution as we noticed that a non negligible proportion of the limb spectra in the CIRS database presented drifts of their continuum toward negative radiances in the $1000-1200 \mathrm{~cm}^{-1}$ spectral region. We removed these spectra from our selections, but we can not exclude that some calibration problems, affecting the intensity of the continuum, remained in our spectra selection. This problem is attenuated at deeper pressure levels and we are therefore more confident in our results below the 0.01-mbar level $(\sim 400 \mathrm{~km})$. Moreover, the signal-to-noise ratio at high altitude can be poor and the derived aerosol extinction can have large error bars. This is particularly true for the Dec. 2009-Jan. 2010 map where the high altitude region appeared strongly depleted (Fig. 6 and 7). It is nevertheless important to notice that, even if aerosol optical depth and temperature profiles are retrieved simultaneously, the limb spectra continuum accuracy has small impact on the derivation of the temperature profile, as the later is inferred from the amplitude of the $\nu_{4} \mathrm{CH}_{4}$ band.

Aerosol mass mixing ratio profiles are derived from the extinction profiles assuming a constant extinction cross section for all altitudes and latitudes, fractal aggregates of 3000 monomers with monomer radius of $0.05 \mu \mathrm{m}$ and a monomer density of $0.6 \mathrm{~g} . \mathrm{cm}^{-3}$ (see Vinatier et al. (2010a) for more details). It represents the mass of aerosols divided by the mass of the air inside a given air parcel.

Fig. 7 shows that aerosols seemed to be enriched at high altitude (above the 0.1 mbar level), excepted on the Dec. 2009 - Jan. 2010 map, for which we believe that the continuum at high altitude is underestimated due to some calibration problems. In the altitude range 
probed by CIRS, we believe that aerosols are good tracers of dynamics as they are formed much higher (at least around 1000 km, (Waite et al., 2005; Coates et al., 2007)) and are probably no more affected by the middle atmosphere chemistry (as they display very similar spectra at several latitudes and altitude levels in the middle atmosphere, see Vinatier et al. (2012)). Then, a descending branch of the global circulation cells should result in aerosol enrichment in the regions of subsidence, bringing the enriched air from higher altitudes to deeper levels. This explains the enrichment observed at high northern latitudes with respect to their southern counterparts at 0.1 mbar between January 2010 and February 2012 (see Figs. 6 and 7).

At the south pole (see Section 4.1.1) a descending branch probably existed in January 2010. Interestingly, the aerosol mass mixing ratio map at the same date displays an enrichment between $85^{\circ} \mathrm{S}$ and $80^{\circ} \mathrm{S}$ in the $0.02-0.001$ mbar region. This very confined enrichment in latitude suggests that the enriched air comes from altitudes higher than $500 \mathrm{~km}$ (pressures lower than 0.001 mbar). In June 2010, this enriched zone extended in latitude and was observed above the 0.01 mbar level down to $70^{\circ} \mathrm{S}$. This latitudinal extension is compatible with a well established downward transport above the south pole since January 2010. At deeper levels, in the 0.01-0.1 mbar region at latitudes higher than $70^{\circ} \mathrm{S}$, a depleted zone was observed in January 2010, which seemed to be transported downwards between June 2010 and February 2012. This is also compatible with the existence of a descending branch there.

We saw from the composition map that a new single circulation cell was probably observed since June 2011 (see Section 4.1.2). The north pole ascending branch should transport the air depleted in aerosols from low altitude towards upper levels. This phenomenon is observed in the Jan.-Feb. 2012 map where the 1-mbar region is depleted between 55 and $75^{\circ} \mathrm{N}$ compared to June 2011.

Another interesting feature is the enriched zone localized between 0.1 and 1 mbar from high southern to mid-northern latitudes, which can be clearly seen on all our maps (except for the map in May 2013 that covers a quite restricted latitudinal region). This enriched zone shows global seasonal variations that are very close to the behavior of the mid-latitude stratopause (see Fig. 2), both regarding its altitude and latitude range. From the present 
study, it is impossible to say if this aerosol enriched zone is responsible for the heating of the stratopause or if the stratopause constitutes a stable dynamically zone, in which aerosols are enriched.

In July and November 2012, we infer a detached haze layer, which is localized at 0.045 mbar $(333 \mathrm{~km})$ in July and 0.06 mbar $(320 \mathrm{~km})$ in November (more details are given in Section 5.2.2).

The depleted zone observed deeper than the 2-mbar level on all maps of Fig. 7 is possibly due to the sedimentation of aerosols that are used as condensing cores for the molecules condensing in Titan's atmosphere (i.e., all molecules except $\mathrm{N}_{2}$, $\mathrm{CO}$ and $\mathrm{C}_{2} \mathrm{H}_{4}$ ).

In January - February 2012, an anomalous depletion is observed at the equator at high altitude (between 0.03 and 0.001 mbar). This region was observed during both months, then during two different flybys, and an analysis of both datasets give similar results. Both datasets could display calibration problems, with maybe underestimated continuum values, but this seems unlikely as all other latitudes do not display any problems. Such a strong depletion is hard to explain, but could be related to some particular dynamical phenomena occurring in the equatorial region. For instance, de Kok et al. (2010) mentioned the existence of an equatorial inhibited mixing region to explain the observation of a tropical haze band seen during the northern winter.

\subsection{Results from the $0.5 \mathrm{~cm}^{-1}$ spectra}

As mentioned in Section 3.2, it was not possible from the $15.5 \mathrm{~cm}^{-1}$ resolution spectra to infer unambiguously the mixing ratios of $\mathrm{C}_{2} \mathrm{H}_{4}, \mathrm{C}_{3} \mathrm{H}_{8}, \mathrm{CH}_{3} \mathrm{C}_{2} \mathrm{H}, \mathrm{C}_{4} \mathrm{H}_{2}, \mathrm{C}_{6} \mathrm{H}_{6}, \mathrm{HC}_{3} \mathrm{~N}$ and $\mathrm{CO}_{2}$. In order to see how the global seasonal changes impacted all molecules observed with CIRS, we performed analysis of some datasets of $0.5 \mathrm{~cm}^{-1}$ resolution limb spectra acquired during the northern spring and compared the results with northern winter observations. From Wilson and Atreya (2004), chemical lifetimes of the above mentionned molecules are higher than 0.8 year around 0.1 mbar (i.e., $\sim 18$ Titan's days), except for $\mathrm{C}_{4} \mathrm{H}_{2}$ and $\mathrm{C}_{6} \mathrm{H}_{6}$ that have chemical lifetimes of 0.04 and 0.015 years (or 0.9 and 0.3 Titan day), respectively. The overturning timescale predicted by the 3-D circulation models of Newman et al. (2011) 
and Lebonnois et al. (2012) is about a few Titan's days. Therefore, we can assume that temporal variations of the vertical mixing ratio profiles of $\mathrm{C}_{2} \mathrm{H}_{4}, \mathrm{C}_{3} \mathrm{H}_{8}, \mathrm{CH}_{3} \mathrm{C}_{2} \mathrm{H}$ and $\mathrm{HC}_{3} \mathrm{~N}$, additionally to $\mathrm{HCN}, \mathrm{C}_{2} \mathrm{H}_{6}$ and $\mathrm{C}_{2} \mathrm{H}_{2}$, are good tracers of the global dynamics.

\subsubsection{High northern latitudes}

The mixing ratio profiles inferred between October 2006 (mid-northern winter) and December 2011 (northern spring) at high northern latitude are displayed in Fig 8. $\mathrm{CO}_{2}$ is the most stable molecule regarding seasonal variations, as it displays no temporal evolutions. $\mathrm{C}_{3} \mathrm{H}_{8}$ also seems to be quite stable, while other molecules present strong variations of the slope of their mixing ratio profiles, particularly after the northern spring equinox. In October 2006 and August 2007, during the northern winter, we do not infer very strong global variations of the mixing ratio profiles, except different oscillating shapes between the two dates for $\mathrm{HC}_{3} \mathrm{~N}, \mathrm{C}_{2} \mathrm{H}_{2}, \mathrm{C}_{4} \mathrm{H}_{2}$ and $\mathrm{HCN}$. After the equinox, in December 2009, all mixing ratio profiles (except $\mathrm{CO}_{2}$ and $\mathrm{C}_{3} \mathrm{H}_{8}$ ) show steeper gradients with an enrichment at high altitude compared to what was observed in 2006 and 2007. This tendency is more accentuated in December 2009 and the molecular mixing ratios reach their maximum values at high altitude in April 2010. This date displayed the most enriched mixing ratios that have ever been seen above Titan's north pole during the Cassini mission. At $0.001 \mathrm{mbar}(\sim 500 \mathrm{~km})$, $\mathrm{C}_{2} \mathrm{H}_{2}$ is enriched by more than a factor of 10 compared to October 2006, HCN is enriched by a factor of $3, \mathrm{C}_{2} \mathrm{H}_{6}$ by a factor of $2, \mathrm{C}_{4} \mathrm{H}_{2}$ by $5, \mathrm{C}_{6} \mathrm{H}_{6}$ by 1000 and $\mathrm{HC}_{3} \mathrm{~N}$ by a factor of $\sim 100$. At 0.005 mbar $(\sim 400 \mathrm{~km}), \mathrm{CH}_{3} \mathrm{C}_{2} \mathrm{H}$ is enriched by a factor of 60 and $\mathrm{C}_{2} \mathrm{H}_{4}$ by a factor of 200. Such high enrichments can be explained by the combination of dynamics and chemistry. From their photochemical model, Lebonnois et al. (2001) showed that at $70^{\circ} \mathrm{N}$, very shortly after the northern spring equinox, an enrichment of molecules is predicted at altitudes higher than $500 \mathrm{~km}$. This enrichment is caused by the increasing insolation above the north pole (which was inside the polar night during winter) at the origin of a reactive photochemistry there. As the circulation is still subsiding above the north pole during the early spring, this enriched air above $500 \mathrm{~km}$ is transported deeper and generates an additional enhancement of molecular gases. It is also interesting to notice that, in April 2010, 
the mixing ratio values at deeper levels (around 1 mbar) were not affected by this strong enrichment and remained comparable to what was observed during the northern winter. One year later, in December 2011, all the mixing ratio vertical gradients were less steep especially above the 0.2 mbar level, which can be explained by the upward motion of depleted air from the deeper stratosphere due to the ascending branch of the new pole-to-pole cell.

Mixing ratio profiles of $\mathrm{C}_{2} \mathrm{H}_{2}, \mathrm{HCN}, \mathrm{C}_{4} \mathrm{H}_{2}$ and $\mathrm{CH}_{3} \mathrm{C}_{2} \mathrm{H}$ often present similar oscillation patterns at a given date. Similar oscillating profiles were observed by Teanby et al. (2009b) at the polar vortex boundary during the northern winter. They mentioned that these smallscale structure could be caused by cross-latitude advection across the vortex boundary.

Fig. 9 shows the seasonal variations of the thermal profile at high northern latitudes for the same dates. Solid lines give levels where the profiles are constrained by CIRS limb spectra. Error bars are $1 \mathrm{~K}$ at maximum. Even if the latitude of these profiles varies from $61^{\circ} \mathrm{N}$ to $88^{\circ} \mathrm{N}$, some global trends with season can be highlighted and are consistent with the scenario of the global dynamics seasonal changes derived from our global maps. In October 2006 and August 2007, a well-marked stratopause is observed around 0.01 mbar $(400 \mathrm{~km})$ with a temperature of about $197 \mathrm{~K}$. Around the northern spring equinox (July and December 2009), the stratopause temperature decreased to $175 \mathrm{~K}$ at $0.03 \mathrm{mbar}(\sim 350 \mathrm{~km})$. Such a temperature decrease of the stratopause is due to the weakening of the descending branch. In April 2010, the stratopause was observed at $0.01 \mathrm{mbar}(400 \mathrm{~km})$ and $175 \mathrm{~K}$, and stratospheric temperatures, from 2 to 0.01 mbar (i.e. 150 to $400 \mathrm{~km}$ ), were colder by about $10 \mathrm{~K}$ compared to what was observed just around the equinox at similar levels. These lower temperatures of the stratosphere could possibly result from the additional radiative cooling caused by the high enhancement of molecular gas, as the weakened descending branch had probably no adiabatic heating impact in this pressure range. In December 2011, as Spring was progressing, the stratopause temperature increased to $179 \mathrm{~K}$ at $\sim 0.025$ mbar $(\sim 350$ $\mathrm{km}$ ) and compensated the probable adiabatic cooling in the same zone due to the ascending branch. 


\subsection{2. $45^{\circ} \mathrm{N}$ and the Equator}

Fig. 10 displays the comparison of the retrieved temperature and mixing ratio profiles at $45^{\circ} \mathrm{N}$ between July 2006 and June 2012. Fig. 11 displays the retrieved temperature and mixing ratio profiles near the equator in January 2007 and May 2012. For both latitudes, the spring observation occurs after the implementation of the new single pole-to-pole cell upwelling at north pole. Stratospheric temperatures in the $0.2-5$ mbar region at $45^{\circ} \mathrm{N}$ and in the 1 - 5 mbar region at the equator remained very similar between winter and spring. At $45^{\circ} \mathrm{N}$, the stratopause temperature is colder by $\sim 5 \mathrm{~K}$ in the spring compared to its winter value, while remaining localized at the same altitude. At the Equator, from winter to spring, we observe a decrease of the altitude of the stratopause moving from 0.1 to 0.5 mbar and a decrease of its temperature from $185 \mathrm{~K}$ to $180 \mathrm{~K}$. Equatorial mesospheric temperatures (above the 0.005 mbar level, $\sim 450 \mathrm{~km}$ ) were noticeably colder (about $20 \mathrm{~K}$ at $0.001 \mathrm{mbar}$, $500 \mathrm{~km}$ ) than the winter values. Colder temperature values are probably related to the lower solar flux in southern spring due to Saturn's orbital eccentricity.

At a given latitude, we notice that the mixing ratio profiles of all molecules are very similar during winter and the spring. Nevertheless, mixing ratio profiles at $45^{\circ} \mathrm{N}$ display more oscillating patterns in spring than during the winter, which may be linked to the dislocation of the northern polar vortex.

\subsection{3. $45^{\circ} S$}

Fig. 12 displays the retrieved temperature and mixing ratio profiles at $45^{\circ} \mathrm{S}$ in December 2007 (southern summer) and February 2012 (southern autumn). Temperatures in 2012 are globally colder than in 2007. During the southern summer, the stratopause was localized at 0.08 mbar $(\sim 300 \mathrm{~km})$ with a temperature of $185 \mathrm{~K}$, and during the autumn its temperature decreased to $174 \mathrm{~K}$ and was localized at a deeper level $(0.3 \mathrm{mbar}, \sim 250 \mathrm{~km})$. As in the equatorial region, mesospheric temperatures in 2012 are colder by about $10 \mathrm{~K}$ at $0.001 \mathrm{mbar}$ than in 2007. Colder temperatures in 2012 at mid-southern latitude are expected as the autumn is progressing.

In February 2012, mixing ratio profiles show steeper gradients above the 0.5 mbar level 
compared to what was observed during summer. $\mathrm{HCN}$ and $\mathrm{C}_{2} \mathrm{H}_{2}$ are particularly enriched at 0.001 mbar $(500 \mathrm{~km})$ by a factor of 10 . At $400 \mathrm{~km}, \mathrm{C}_{2} \mathrm{H}_{6}$ is enriched by a factor of 2 at $0.01 \mathrm{mbar}(400 \mathrm{~km})$. This enrichment is explained by the descending branch in place above the south pole since January 2010 during the southern autumn and transporting enriched air from higher levels. These observed enrichments are in agreement with those derived from $15.5 \mathrm{~cm}^{-1}$ resolution limb spectra (see Section 4.1 .2 ).

\subsubsection{High southern latitudes}

Figure 13 displays the temporal evolution of temperature and molecular mixing ratio profiles at high southern latitudes during the southern autumn between January 2010 and September 2011.

In January 2010, the stratopause is localized at $\sim 0.1 \mathrm{mbar}(\sim 270 \mathrm{~km})$ for a maximum temperature of $183 \mathrm{~K}$, while its altitude increased in September 2011 to reach 0.01 mbar (400 km) with a temperature of $177 \mathrm{~K}$. In the same time, the stratospheric temperature at 1 mbar decreased by about $15 \mathrm{~K}$. The colder temperatures around 1 mbar can be explained by the lower insolation there while autumn progressed and the high altitude of the stratopause can be explained by the adiabatic heating warming more efficiently this high level region. This observation is in agreement with the results derived from the retrievals of the 15.5 cm-1 limb spectra (see the thermal map of May-June 2011 in Fig. 2).

Molecular mixing ratios present strong modifications of their vertical gradients between both dates, particularly above the 0.02 mbar level, where the mixing ratios of $\mathrm{HCN}, \mathrm{HC}_{3} \mathrm{~N}$, $\mathrm{C}_{2} \mathrm{H}_{4}, \mathrm{C}_{4} \mathrm{H}_{2}, \mathrm{CH}_{3} \mathrm{C}_{2} \mathrm{H}$ and $\mathrm{C}_{6} \mathrm{H}_{6}$ all gained a factor of 500 to 1000 between the $0.02 \mathrm{mbar}$ ( $365 \mathrm{~km})$ and $0.001 \mathrm{mbar}(500 \mathrm{~km})$ levels. This confirms the weel-esthablished subsiding branch inferred from the global maps of $\mathrm{C}_{2} \mathrm{H}_{2}$ and $\mathrm{HCN}$ in June 2011. A local minimum mixing ratio is observed for all these molecules around 0.03 - 0.05 mbar, while at 1 mbar, we observe the same mixing ratio values between January 2010 and September 2011. Such high enhancements are due to the south pole descending branch. Temporal evolution of the depth of this enhanced zone can give us some information on the subsidence speed in the mesosphere (see Section 5.1). 


\section{Discussion}

\subsection{Seasonal evolution of the global dynamics derived from temperature and mixing ratio distributions}

Results regarding the spatial and vertical distributions inferred in the present study can be interpreted as follows. During the northern winter, the descending branch of the single circulation cell generated an adiabatic heating in the 0.001-0.1 mbar region, which resulted in the very high temperatures (up to $206 \mathrm{~K}$ ) observed above the north pole. Later, as the global dynamics was changing and the two circulation cells were in competition, the downwelling at the north pole weakened, and accordingly did the adiabatic heating. For the first time in January 2010, we observe a warming of the mesosphere above the south pole, which suggests that the descending branch existed in this region and created an adiabatic heating there. The observed aerosol enrichment at $85^{\circ} \mathrm{S}$ certainly resulted also from this subsidence but molecular mixing ratios were not yet enriched at this date. Simultaneously, quite high temperatures were still observed in the same pressure range above the north pole and molecules were highly enriched there compared to before. This suggests that a descending branch was still occuring at the north pole. We therefore believe that two circulation cells existed simultaneously in Dec. 2009 and Jan. 2010 with their descending branches above each pole. This dynamical pattern remained up to at least June 2010, where mesospheric south pole temperature increased and the north pole enriched zone was observed deeper compared to January 2010. The solar longitude $\left(\mathrm{L}_{s}\right)$ corresponding to June 2010 is $10^{\circ}$, which is in agreement with the general circulation model predictions of Hourdin et al. (2004), who predicted a two circulation cell regime in the $\mathrm{L}_{s}=350-15^{\circ}$ range.

The upwelling branch of both cells should create a decrease of the temperature by adiabatic cooling. But because of the weakening of the global dynamics while the two circulation cells co-exist (Lebonnois et al., 2012; Newman et al., 2011), General Circulation Models predict quite small temperature decrease by adiabatic cooling (typically lower than $5 \mathrm{~K}$, Newman et al. (2011)) at high altitude in the upwelling branch of the cells. This is quite difficult to observe here from our global maps. 
One year later, in June 2011, temperature above the south pole continued to increase, while $\mathrm{C}_{2} \mathrm{H}_{2}$ and $\mathrm{HCN}$ mixing ratios were enriched at high altitude, as it would be expected with the presence of the descending branch there. Simultaneously, enrichment of $\mathrm{C}_{2} \mathrm{H}_{2}$, $\mathrm{HCN}$ and $\mathrm{C}_{2} \mathrm{H}_{6}$ was observed at mid-northern latitudes and high altitude. This enrichment reinforced in January 2012 in the same region. This would suggest that the enriched air that was confined inside the north polar vortex during winter was advected towards the equator by the upper branch of the new pole-to-pole cell. It is therefore possible that the global circulation had totally reversed already in June 2011 with an upwelling branch at the north pole and a downwelling branch at the south pole. This one-cell new circulation pattern should remain up to the northern autumn equinox in 2025. This scenario is confirmed by our later observations as, in December $2011\left(\mathrm{~L}_{s}=28^{\circ}\right)$, all the mixing ratios at the north pole (Fig. 8) display a depletion at high altitude, which is consistent with the upwelling branch bringing depleted air from the lower stratosphere where a sink of molecules exist due to their condensation. Simultaneously, in January 2012, aerosol mass mixing ratio distribution displayed a depletion in the lower stratosphere, which is also in agreement with the presence of upwelling depleted air there. In June 2012 (at $45^{\circ} \mathrm{N}$, see Fig. 10) and July 2012, the enrichment observed at high altitude and mid-northern latitudes is not observed. This observation seems to be real as it results from the analysis of two different datasets. It is maybe related to the oscillating pattern that is obvious on the profiles of Fig. 10. In November 2012 and May 2013, we observe an enrichment of all molecules at high altitude down to $40^{\circ} \mathrm{S}$ in November 2012 and $25^{\circ} \mathrm{S}$ in May 2013. This suggests that the enriched zone observed right above the south pole in February 2012 has extended later in autumn season towards lower latitudes.

It is possible to derive the speed of the air subsiding at the south pole by looking at the temporal evolution of the location of the molecular enriched zone. Fig. 14 displays the temporal evolution of the mixing ratio profiles of $\mathrm{HCN}$ and $\mathrm{C}_{2} \mathrm{H}_{2}$ during southern autumn, between January 2010 and February 2012, for latitudes higher than 80S. At high altitude, above the 0.01 mbar level, these molecules showed strong enrichments that increased while season was progressing. This enrichment was first observed by Teanby et al. (2012), who 
analyzed CIRS limb data in the south polar region up to September 2011. Assuming that this molecular enhancement was due to air downwelling, they derived vertical speed between 0.8 and $2.3 \mathrm{~mm} / \mathrm{s}$ from the observed enrichment at $450 \mathrm{~km}$ in June 2011 compared to the observed values in January 2010. From the present analysis, we can estimate the speed of this subsidence directly by looking at the altitude evolution of a given molecular mixing ratio value. This calculation assumes that vertical advection dominates over both photochemistry (i.e. dynamical timescales are shorter than the chemical timescale) and horizontal advection. Around $450 \mathrm{~km}$, we derive from the HCN mixing ratio profiles a downward vertical velocity of $4.4 \mathrm{~mm} . \mathrm{s}^{-1}$ in the periods of June - Sept. 2011 and Sept. 2011 - Feb. 2012 (from the altitude variations of the $6.5 \times 10^{-6}$ and $3.5 \times 10^{-5}$ mixing ratio value, respectively). At the same altitude, from the $\mathrm{C}_{2} \mathrm{H}_{2}$ mixing ratio profiles, we derive a vertical velocity of 12.0 mm.s $\mathrm{s}^{-1}$ in the June - Sept. 2011 period (using the $6.6 \times 10^{-6}$ mixing ratio value) and 5.6 mm.s ${ }^{-1}$ in the Sept. 2011 - Feb. 2012 period (using the $3.1 \times 10^{-5}$ mixing ratio value). We believe that the vertical velocity derived from the HCN mixing ratio profiles enrichment is more accurate than the one derived from $\mathrm{C}_{2} \mathrm{H}_{2}$ because $\mathrm{HCN}$ enrichment is more pronounced at high altitude and displays a very steep gradient that is also observed for $\mathrm{C}_{2} \mathrm{H}_{4}, \mathrm{CH}_{3} \mathrm{C}_{2} \mathrm{H}$, $\mathrm{C}_{4} \mathrm{H}_{2}, \mathrm{HC}_{3} \mathrm{~N}$ and $\mathrm{C}_{6} \mathrm{H}_{6}$ (see Fig. 14), while $\mathrm{C}_{2} \mathrm{H}_{2}$ displays a weaker vertical gradient. The velocity of the subsidence that we derive here from $\mathrm{HCN}$ mixing ratio is slightly higher than what was derived by Teanby et al. (2012) earlier in the season, which may suggest an increase of the downward velocity while autumn is progressing, as predicted by General Circulation Models.

At $0.01 \mathrm{mbar}(380 \mathrm{~km})$, where a temperature increase is observed from December 2009 to June 2011 (see Fig. 2), we derive a downward velocity of $2.5 \mathrm{~mm} . \mathrm{s}^{-1}$ in the periods of June - Sept. 2011 and Sept. 2011-Feb. 2012 (from the $4.4 \times 10^{-7}$ and $2.9 \times 10^{-6} \mathrm{HCN}$ mixing ratio values, respectively). From this vertical velocity value, we can derive the increase of the temperature due to adiabatic heating from Eq. 5 of Achterberg et al. (2011). The resulting temperature is given by: 


$$
T=T_{r}-w \tau_{r}\left(\frac{\partial T}{\partial z}-\Gamma\right)
$$

where $\mathrm{T}_{r}$ is the radiative temperature (estimated to be equal to $\sim 173 \mathrm{~K}$ outside the subsiding air region, between $40^{\circ} \mathrm{S}$ and $60^{\circ} \mathrm{S}$ at the same pressure level, which is in agreement with radiative calculations of Hourdin et al. (1995) at $0.01 \mathrm{mbar}$ and $85^{\circ} \mathrm{S}$ ), $w$ is the vertical velocity $\left(w=-2.5 \mathrm{~mm} . \mathrm{s}^{-1}\right), \Gamma$ is the dry adiabatic lapse rate $\left(\Gamma=-1.15 \mathrm{~K} . \mathrm{km}^{-1}\right.$, from Flasar et al. $(2005)), \tau_{r}$ is the radiative relaxation time $\left(\tau_{r} \sim 3 \times 10^{6}\right.$ s from Fig. 14 of Achterberg et al. (2011)), and $z$ is the altitude. We measure $\frac{\partial T}{\partial z}=0.08 \mathrm{~K} . \mathrm{km}^{-1}$. The calculated temperature is then equal to $182 \mathrm{~K}$, which is in good agreement with the measured temperature of $184 \mathrm{~K}$. This vertical velocity is expected to increase as the southern winter approaches, and should be finally comparable to what was observed at the north pole during the northern winter, where the air downwelling had a vertical velocity of about $10 \mathrm{~cm} . \mathrm{s}^{-1}$ (Achterberg et al., 2011), generating temperatures as high as $~ 200 \mathrm{~K}$ at $0.01 \mathrm{mbar}$.

In the Sept. 2011 - Feb. 2012 period, we measure a vertical velocity similar to the one measured in June 2011. In a first approximation, if the atmospheric composition remained the same between both periods, we would expect comparable temperature above the south pole as the vertical downwelling is of similar strength. But instead, we observe much cooler temperature $\left(157 \mathrm{~K}\right.$ at $0.01 \mathrm{mbar}$ at $\left.82^{\circ} \mathrm{S}\right)$ in February 2012. At the same time, a strong enrichment of radiative active trace gases $\left(\mathrm{C}_{2} \mathrm{H}_{2}, \mathrm{C}_{2} \mathrm{H}_{4}, \mathrm{C}_{2} \mathrm{H}_{6}, \mathrm{C}_{3} \mathrm{H}_{4}, \mathrm{C}_{4} \mathrm{H}_{2}, \mathrm{C}_{6} \mathrm{H}_{6}, \mathrm{HCN}\right.$ and $\mathrm{HC}_{3} \mathrm{~N}$ ) occurred above the 0.02 mbar level (see Figs. 3, 4, 5 and 13). These molecules, which are radiative coolers, may have cooled so much the high atmosphere that it compensated and exceeded the adiabatic heating of the subsiding air. Precise calculations would be needed to quantify this effect.

\subsection{Aerosol spatial distribution}

\subsubsection{Polar regions}

The south pole region experienced strong seasonal variations after the 2009 August equinox. As soon as January 2010, an enrichment of aerosol was observed right above the south pole (see Fig. 6 and 7) in the 0.01-0.001 mbar region and was confined at lat- 
itudes higher than $80^{\circ} \mathrm{S}$. Such a confinement above the south pole would suggest that the aerosol enriched air came from much higher levels. In June 2010, this high altitude southern enrichment was more pronounced, and extended toward $70^{\circ} \mathrm{S}$, suggesting the reinforcement of the descending branch there.

Nevertheless, above the south pole, in February 2012, November 2012 and May 2013, we observe an anti-correlation between the aerosol spatial distribution and the molecular mixing ratios. While a strong enhanced region in $\mathrm{C}_{2} \mathrm{H}_{2}, \mathrm{HCN}$ and $\mathrm{C}_{2} \mathrm{H}_{6}$ is observed just above $80^{\circ} \mathrm{S}$ in February 2012 at high altitude and extended towards mid southern latitudes later, a depleted region of aerosols is observed at the same time with similar horizontal motion towards mid latitudes. As aerosols are formed at high altitude, the subsidence at the south pole should create an enrichment and not a sudden depletion. This observation is quite puzzling and more observations of the south pole are needed to verify if this aerosol depletion is real and interpret it.

\subsubsection{Equatorial region}

Fig. 15 shows several extinction vertical profiles extracted from the maps of June 2010, June 2011, July 2012 and November 2012 in the mid-latitude and equatorial regions. The 1- $\sigma$ error bars are plotted at the line-of-sight altitudes of the limb spectra used to retrieve the extinction profiles. These northern spring profiles are compared with a mean profile at $5^{\circ} \mathrm{N}$ derived from CIRS limb spectra during the northern winter in January 2007 (adapted from Fig. 5 of Vinatier et al. (2010a), where the profile was scaled by a factor of 4.5 in order to take into account the spectral variation of aerosol extinction between 610 and 1090 $\left.\mathrm{cm}^{-1}\right)$.

At mid-latitudes and in the equatorial region, Figs 6, 7 and 15 show that aerosols do not seem to undergo seasonal variations below the $\sim 0.3$ mbar level $(\sim 250 \mathrm{~km})$. In contrast, we observe after the equinox, the presence of a single extinction minimum localized around 350 km in June 2010 and June 2011 and two local minima around 300 and $400 \mathrm{~km}$ in July and November 2012.

Our extinction profiles can be compared with those inferred by West et al. (2011) from 
the visible images acquired by Cassini/ISS. For an easier comparison, Fig. 15 utilizes the same vertical scale as Fig. 3 of West et al. (2011). Their ISS observations were performed at $343 \mathrm{~nm}$, while ours are representative of the $9.2 \mu \mathrm{m}$ wavelength $\left(1090 \mathrm{~cm}^{-1}\right)$. In order to quantitatively compare our extinction values with those of West et al. (2011), we must apply a scaling factor to our values. To do so, we used the spectral dependence of the aerosol extinction cross section that we inferred in a previous study (Fig. 2 of Vinatier et al. (2012)). This spectral dependence was derived from the results of Tomasko et al. (2008) based on the in situ Huygens/DISR and from CIRS observations. At $343 \mathrm{~nm}$ (i.e. 29150 $\mathrm{cm}^{-1}$ ), an extrapolation of the DISR values gives an extinction cross section of $4.0 \times 10^{-7}$ $\mathrm{cm}^{2}$, while this cross section is of $4.8 \times 10^{-10} \mathrm{~cm}^{2}$ at $1090 \mathrm{~cm}^{-1}$. Then a factor of about 835 must be applied to our values at $1090 \mathrm{~cm}^{-1}$ in order to compare them with the West et al. ones at $343 \mathrm{~nm}$. At $200 \mathrm{~km}$, our June 2010 and June 2011 extinction coefficients would then correspond to a value of $3.3 \times 10^{-7} \mathrm{~cm}^{-1}$, or $3.3 \times 10^{-2} \mathrm{~km}^{-1}$ at $343 \mathrm{~nm}$, which is in good agreement with West et al. (2011) results, who inferred $2.2 \times 10^{-2} \mathrm{~km}^{-1}$ in April 2010 at the same altitude. In June 2010, we derive a minimum of the aerosol extinction profiles localized around $330-350 \mathrm{~km}$, which is much less pronounced than the minimum inferred by West et al. (2011) in April 2010. They derived a minimum extinction at $330 \mathrm{~km}$ of $6 \times 10^{-6} \mathrm{~km}^{-1}$, while our mean minimum value is $\sim 1 \times 10^{-11} \mathrm{~cm}^{-1}$ at $1090 \mathrm{~cm}^{-1}$, which would correspond to $8.4 \times 10^{-4} \mathrm{~km}^{-1}$ at $343 \mathrm{~nm}$, which is 140 times higher than their results. Such differences can only partly be explained by the lower vertical resolution of CIRS ( 30 $\mathrm{km}$ ) compared to ISS (resolution of $\sim 10 \mathrm{~km}$ ). At $400 \mathrm{~km}$, in June 2010, we derive a mean value of $8.4 \times 10^{-12} \mathrm{~cm}^{-1}$ at $1090 \mathrm{~cm}^{-1}$, corresponding to $7.0 \times 10^{-4} \mathrm{~km}^{-1}$ at $343 \mathrm{~nm}$, which is higher than the West et al. value by a factor of 3. In June 2011, we observe a more pronounced minimum extinction at $\sim 340 \mathrm{~km}$.

In July 2012 and November 2012, we inferred a distinct detached haze layer on the midlatitude extinction profiles at 330 and $320 \mathrm{~km}$, respectively. At altitudes higher than 450 $\mathrm{km}$, the extinction coefficient is higher than what we observed during the northern winter, while below this altitude, it was lower than the winter values. West et al. (2013) mentioned that they inferred a decrease of the altitude of the detached haze layer with time after the 
northern spring equinox. Actually, their analysis of ISS images shows that the appearance of a detached layer is produced by a gap in the haze vertical profile and it is the gap rather than the haze layer that drops in altitude. If we focus on the altitude of the gap below the detached haze layer, in June 2011 we infer a minimum of the extinction localized at 340 $\mathrm{km}$, which is comparable to the altitude of the gap observed in ISS images acquired in April 2011 (West et al., 2013). There is therefore an agreement between the aerosol extinction derived from the Cassini/ISS UV images and the extinction derived from the mid-infrared CIRS observations.

\section{Conclusions}

From this study of the spatial distribution of temperature, aerosol and molecular mixing ratios derived from CIRS limb spectra, we can now have some constraints on the timing of the reversal of the global dynamics in the middle atmosphere of Titan that occurred just after the 2009 August 11 northern spring equinox:

1. In January 2010, a descending branch was probably already occurring above the south pole and was at the origin of the aerosol enrichment confined to latitudes higher than $80^{\circ} \mathrm{S}$. This subsidence, generating an adiabatic heating, was probably also at the origin of the second local temperature maxima observed at 0.005 mbar, which was not observed during the southern summer in 2008 (Achterberg et al., 2011). Moreover, as north pole high altitude temperature was still high $(180 \mathrm{~K})$ and the molecular mixing ratios were highly enriched compared to other latitudes, there is strong indication that air downwelling still occurred there. Then, in January 2010 ( 10 Titan's days after the equinox), Titan's middle atmosphere probably had already transitionned to a two cell circulation regime with downward motion at both poles. The timing is consistent with radiative time constant for Titan's middle atmosphere (0.02 Titan's year, or 13 Titan's days). We are not able to infer where the ascending branch occurred from our observations.

2. In April and June 2010, as very high enrichments of all molecules were observed (see Fig. 8) above the north pole at high altitude, the descending branch was probably still existing at this time at the north pole. Simultaneously, temperature above the south pole 
increased at high altitude, due to the adiabatic heating of the air subsiding there. Therefore, a two circulation cell regime still existed in June 2010.

3. In June 2011, enrichment of $\mathrm{HCN}$ and $\mathrm{C}_{2} \mathrm{H}_{2}$ is observed right above the south pole above the 0.004 mbar level (Figs. 3, 4). This enriched zone subsequently extended towards deeper levels and reached the 0.03 mbar level in February 2012. During this June 2011-February 2012 period, we derive a constant downwards velocity of $2.5 \mathrm{~mm} / \mathrm{s}$ above the south pole at $0.01 \mathrm{mbar}(400 \mathrm{~km})$. Simultaneously, at mid-northern latitudes, an enrichment at high altitude (above the 0.03 mbar level) appeared and subsequently extended toward the equator. It apprently originated from the enriched zone above the north pole, which was localized inside the polar vortex during winter. This mid-northern latitude air enrichment was more pronounced in January 2012. This suggests that the global dynamics had totally reversed since at least June 2011, with a single pole-to-pole cell occurring with an ascending branch at the north pole bringing the enriched air accumulated during the northern winter towards mid latitudes at high altitude. This is also corroborated with the vertical distribution of the mixing ratios derived in December 2011 (Fig. 8) at the north pole, which did not show anymore the very high enhancement in the 0.1-0.001 mbar range that was observed earlier. The upwelling of depleted air from low altitudes to higher altitudes can be responsible for this molecular gas depletion between April 2010 and December 2011. Therefore, there are several arguments that support a transition between two circulation cells and one single cell occurring between June 2010 and June 2011.

4. The altitude of the mid-latitude stratopause was observed deeper a few months before the northern spring equinox, in May 2009, than during the winter (Achterberg et al., 2011). In the mid-northern winter, the stratopause was observed at 0.07 mbar $(320 \mathrm{~km})$ with a temperature of $185 \mathrm{~K}$, while since May 2009, its altitude and temperature gradually decreased from 0.02 mbar $(270 \mathrm{~km})$ and $\sim 183 \mathrm{~K}$ at 0.5 mbar $(215 \mathrm{~km})$ with $177 \mathrm{~K}$ in May 2013. This seasonal evolution could be related to the reduced solar flux at Titan between northern winter and mid-spring because of Saturn's orbital eccentricity.

5. The observed molecular gas enriched zone above the south pole, which appeared in June 2011, seemed to expand towards lower latitudes, with southern autumn progressing, 
while staying localized at high altitude above the 0.02 mbar level, and was observed down to $25^{\circ} \mathrm{S}$ in May 2013.

6. We observed a detached haze layer in July and November 2012 localized at similar altitudes as seen by the Cassini/ISS instrument.

Thanks to CIRS observations, we were able here to put timing constraints on midatmosphere dynamical reversal after the northern spring equinox. A more detailed study will need the help of 3D General Circulation Models in particular to decouple the radiative impact of emitters to the adiabatic effects due to vertical motions of air. With the Cassini mission extension up to 2017, half a Titan's year will have passed and it will be very interesting to continue to monitor the temporal evolution of the spatial distributions of temperature, molecules and aerosol especially to see how the unexpected phenomena observed above the south pole up to now evolve during the southern autumn.

The vertical profiles that were presented in this study will be posted on a database hosted at the OV-Paris Data Centre and will then be available for all the community.

\section{Acknowledgments}

This work was funded by the Centre National d'Etudes Spatiales, the Programme National de Planétologie (INSU), the Agence Nationale de la Recherche (ANR 2011 Blanc SIMI 5-6 002-01"APOSTIC'), and The Leverhulme Trust and Science and Technology Facilities Council and the NASA Cassini poject. We thank Darrell Strobel and an anonymous reviewer for their suggestions to improve this paper.

\section{References}

Achterberg, R. K., Conrath, B. J., Gierasch, P. J., Flasar, F. M., Nixon, C. A., Mar. 2008. Titan's middleatmospheric temperatures and dynamics observed by the Cassini Composite Infrared Spectrometer. Icarus $194,263-277$.

Achterberg, R. K., Gierasch, P. J., Conrath, B. J., Michael Flasar, F., Nixon, C. A., Jan. 2011. Temporal variations of Titan's middle-atmospheric temperatures from 2004 to 2009 observed by Cassini/CIRS. Icarus 211, 686-698. 
Bézard, B., Nov. 2014. The methane mole fraction in Titan's stratosphere from DISR measurements during the Huygens probe's descent. Icarus 242, 64-73.

Coates, A. J., Crary, F. J., Lewis, G. R., Young, D. T., Waite, J. H., Sittler, E. C., Nov. 2007. Discovery of heavy negative ions in Titan's ionosphere. Geophys. Res. Lett. 34, L22103.

Conrath, B. J., Gierasch, P. J., Ustinov, E. A., Oct. 1998. Thermal Structure and Para Hydrogen Fraction on the Outer Planets from Voyager IRIS Measurements. Icarus 135, 501-517.

Coustenis, A., Achterberg, R. K., Conrath, B. J., Jennings, D. E., Marten, A., Gautier, D., Nixon, C. A., Flasar, F. M., Teanby, N. A., Bézard, B., Samuelson, R. E., Carlson, R. C., Lellouch, E., Bjoraker, G. L., Romani, P. N., Taylor, F. W., Irwin, P. G. J., Fouchet, T., Hubet, A., Orton, G. S., Kunde, V. G., Vinatier, S., Mondellini, J., Abbas, M. M., Courtin, R., Jun. 2007. The composition of Titan's stratosphere from Cassini/CIRS mid-infrared spectra. Icarus 189, 35-62, doi:10.1016/j.icarus.2006.12.022. de Kok, R., Irwin, P. G. J., Teanby, N. A., Vinatier, S., Tosi, F., Negrão, A., Osprey, S., Adriani, A., Moriconi, M. L., Coradini, A., May 2010. A tropical haze band in Titan's stratosphere. Icarus 207, 485-490.

de Kok, R. J., Teanby, N. A., Maltagliati, L., Irwin, P. G. J., Vinatier, S., Oct. 2014. HCN ice in Titan's high-altitude southern polar cloud. Nature 514, 65-67.

Flasar, F. M., Achterberg, R. K., Conrath, B. J., Gierasch, P. J., Kunde, V. G., Nixon, C. A., Bjoraker, G. L., Jennings, D. E., Romani, P. N., Simon-Miller, A. A., Bézard, B., Coustenis, A., Irwin, P. G. J., Teanby, N. A., Brasunas, J., Pearl, J. C., Segura, M. E., Carlson, R. C., Mamoutkine, A., Schinder, P. J., Barucci, A., Courtin, R., Fouchet, T., Gautier, D., Lellouch, E., Marten, A., Prangé, R., Vinatier, S., Strobel, D. F., Calcutt, S. B., Read, P. L., Taylor, F. W., Bowles, N., Samuelson, R. E., Orton, G. S., Spilker, L. J., Owen, T. C., Spencer, J. R., Showalter, M. R., Ferrari, C., Abbas, M. M., Raulin, F., Edgington, S., Ade, P., Wishnow, E. H., May 2005. Titan's Atmospheric Temperatures, Winds, and Composition. Science 308, 975-978.

Flasar, F. M., Conrath, B. J., Jun. 1990. Titan's stratospheric temperatures - A case for dynamical inertia? Icarus 85, 346-354.

Flasar, F. M., Kunde, V. G., Abbas, M. M., Achterberg, R. K., Ade, P., Barucci, A., Bézard, B., Bjoraker, G. L., Brasunas, J. C., Calcutt, S., Carlson, R., C'esarsky, C. J., Conrath, B. J., Coradini, A., Courtin, R., Coustenis, A., Edberg, S., Edgington, S., Ferrari, C., Fouchet, T., Gautier, D., Gierasch, P. J., Grossman, K., Irwin, P., Jennings, D. E., Lellouch, E., Mamoutkine, A. A., Marten, A., Meyer, J. P., Nixon, C. A., Orton, G. S., Owen, T. C., Pearl, J. C., Prangé, R., Raulin, F., Read, P. L., Romani, P. N., Samuelson, R. E., Segura, M. E., Showalter, M. R., Simon-Miller, A. A., Smith, M. D., Spencer, J. R., Spilker, L. J., Taylor, F. W., Dec. 2004. Exploring The Saturn System In The Thermal Infrared: The Composite Infrared Spectrometer. Space Sci. Rev. 115, 169-297. 
Fulchignoni, M., Ferri, F., Angrilli, F., Ball, A. J., Bar-Nun, A., Barucci, M. A., Bettanini, C., Bianchini, G., Borucki, W., Colombatti, G., Coradini, M., Coustenis, A., Debei, S., Falkner, P., Fanti, G., Flamini, E., Gaborit, V., Grard, R., Hamelin, M., Harri, A. M., Hathi, B., Jernej, I., Leese, M. R., Lehto, A., Lion Stoppato, P. F., López-Moreno, J. J., Mäkinen, T., McDonnell, J. A. M., McKay, C. P., Molina-Cuberos, G., Neubauer, F. M., Pirronello, V., Rodrigo, R., Saggin, B., Schwingenschuh, K., Seiff, A., Simões, F., Svedhem, H., Tokano, T., Towner, M. C., Trautner, R., Withers, P., Zarnecki, J. C., Dec. 2005. In situ measurements of the physical characteristics of Titan's environment. Nature 438, 785-791.

Hourdin, F., Lebonnois, S., Luz, D., Rannou, P., Dec. 2004. Titan's stratospheric composition driven by condensation and dynamics. J. Geophys. Res. 109, E12005, doi:10.1029/2004JE002282.

Hourdin, F., Talagrand, O., Sadourny, R., Courtin, R., Gautier, D., McKay, C. P., Oct. 1995. Numerical simulation of the general circulation of the atmosphere of Titan. Icarus 117, 358-374.

Jennings, D. E., Anderson, C. M., Samuelson, R. E., Flasar, F. M., Nixon, C. A., Bjoraker, G. L., Romani, P. N., Achterberg, R. K., Cottini, V., Hesman, B. E., Kunde, V. G., Carlson, R. C., de Kok, R., Coustenis, A., Vinatier, S., Bampasidis, G., Teanby, N. A., Calcutt, S. B., Dec. 2012. First Observation in the South of Titan's Far-infrared $220 \mathrm{~cm}^{-1}$ Cloud. Astrophy. J. 761, L15.

Jolly, A., Fayt, A., Benilan, Y., Jacquemart, D., Nixon, C. A., Jennings, D. E., May 2010. The $\nu_{8}$ Bending Mode of Diacetylene: From Laboratory Spectroscopy to the Detection of ${ }^{13} \mathrm{C}$ Isotopologues in Titan's Atmosphere. Astrophys. J. 714, 852-859.

Jolly, A., Manceron, L., Kwabia-Tchana, F., Benilan, Y., Gazeau, M.-C., 2014. Revised infrared bending mode intensities for diacetylene $\left(\mathrm{C}_{4} \mathrm{H}_{2}\right)$ : application to Titan. Planet. Space. Sci., accepted.

Khlifi, M., Paillous, P., Delpech, C., Nishio, M., Bruston, P., Raulin, F., Nov. 1995. Absolute IR band intensities of diacetylene in the $250-4300 \mathrm{~cm}^{-1}$ region: implications for Titan's atmosphere. J. Molec. Spec. 174, 116-122.

Kunde, V. G., Ade, P. A., Barney, R. D., Bergman, D., Bonnal, J.-F., Borelli, R., Boyd, D., Brasunas, J. C., Brown, G., Calcutt, S. B., Carroll, F., Courtin, R., Cretolle, J., Crooke, J. A., Davis, M. A., Edberg, S., Fettig, R., Flasar, M., Glenar, D. A., Graham, S., Hagopian, J. G., Hakun, C. F., Hayes, P. A., Herath, L., Horn, L., Jennings, D. E., Karpati, G., Kellebenz, C., Lakew, B., Lindsay, J., Lohr, J., Lyons, J. J., Martineau, R. J., Martino, A. J., Matsumura, M., McCloskey, J., Melak, T., Michel, G., Morell, A., Mosier, C., Pack, L., Plants, M., Robinson, D., Rodriguez, L., Romani, P., Schaefer, W. J., Schmidt, S., Trujillo, C., Vellacott, T., Wagner, K., Yun, D., Oct. 1996. Cassini infrared Fourier spectroscopic investigation. In: Horn, L. (Ed.), Cassini/Huygens: A Mission to the Saturnian System, Proc. SPIE Vol. 2803. pp. $162-177$.

Lavvas, P., Sander, M., Kraft, M., Imanaka, H., Feb. 2011. Surface Chemistry and Particle Shape: Processes for the Evolution of Aerosols in Titan's Atmosphere. Astrophys. J. 728, 80. 
Lebonnois, S., Burgalat, J., Rannou, P., Charnay, B., Mar. 2012. Titan global climate model: A new 3-dimensional version of the IPSL Titan GCM. Icarus 218, 707-722.

Lebonnois, S., Toublanc, D., Hourdin, F., Rannou, P., Aug. 2001. Seasonal variations of Titan's atmospheric composition. Icarus 152, 384-406.

Lellouch, E., Bézard, B., Flasar, F. M., Vinatier, S., Achterberg, R., Nixon, C. A., Bjoraker, G. L., Gorius, N., Mar. 2014. The distribution of methane in Titan's stratosphere from Cassini/CIRS observations. Icarus 231, 323-337.

Maltagliati, L., Bézard, B., Vinatier, S., Hedman, M., Lellouch, M., Nicholson, P. D., Sotin, C., deKok, R., Sicardy, B., 2014. Titan's atmosphere as observed by VIMS/Cassini solar occultations: gaseous components. submitted.

Newman, C. E., Lee, C., Lian, Y., Richardson, M. I., Toigo, A. D., Jun. 2011. Stratospheric superrotation in the TitanWRF model. Icarus 213, 636-654.

Niemann, H. B., Atreya, S. K., Demick, J. E., Gautier, D., Haberman, J. A., Harpold, D. N., Kasprzak, W. T., Lunine, J. I., Owen, T. C., Raulin, F., Dec. 2010. Composition of Titan's lower atmosphere and simple surface volatiles as measured by the Cassini-Huygens probe gas chromatograph mass spectrometer experiment. Journal of Geophysical Research (Planets) 115, 12006.

Rannou, P., Cours, T., Le Mouélic, S., Rodriguez, S., Sotin, C., Drossart, P., Brown, R., Aug. 2010. Titan haze distribution and optical properties retrieved from recent observations. Icarus 208, 850-867.

Strobel, D. F., Atreya, S. K., Bézard, B., Ferri, F., Flasar, F. M., Fulchignoni, M., Lellouch, E., MüllerWodarg, I., 2010. Atmospheric Structure and Composition. p. 235.

Teanby, N. A., de Kok, R., Irwin, P. G. J., Osprey, S., Vinatier, S., Gierasch, P. J., Read, P. L., Flasar, F. M., Conrath, B. J., Achterberg, R. K., Bézard, B., Nixon, C. A., Calcutt, S. B., Dec. 2008b. Titan's winter polar vortex structure revealed by chemical tracers. J. Geophys. Res. 113, E12003, doi: 10.1029/2008JE003218.

Teanby, N. A., Irwin, P. G. J., de Kok, R., Irwin, P. G. J., Dec. 2009b. Small scale composition and haze layering in Titan's polar vortex. Icarus 204, 645-657.

Teanby, N. A., Irwin, P. G. J., de Kok, R., Nixon, C. A., Feb. 2009a. Dynamical implications of seasonal and spatial variations in Titan's stratospheric composition. Phil. Trans. R. Soc. A 367, 697-711.

Teanby, N. A., Irwin, P. G. J., de Kok, R., Nixon, C. A., Coustenis, A., Bézard, B., Calcutt, S. B., Bowles, N. E., Flasar, F. M., Fletcher, L., Howett, C., Taylor, F. W., 2006. Latitudinal variations of HCN, $\mathrm{HC}_{3} \mathrm{~N}$, and $\mathrm{C}_{2} \mathrm{~N}_{2}$ in Titan's stratosphere derived from Cassini CIRS data. Icarus 181, 243-255.

Teanby, N. A., Irwin, P. G. J., de Kok, R., Vinatier, S., Bézard, B., Nixon, C. A., Flasar, F. M., Calcutt, S. B., Bowles, N. E., Fletcher, L., Howett, C., Taylor, F. W., Feb. 2007. Vertical profiles of HCN, HC 3 N, and $\mathrm{C}_{2} \mathrm{H}_{2}$ in Titan's atmosphere derived from Cassini/CIRS data. Icarus 186, 364-384. 
Teanby, N. A., Irwin, P. G. J., Nixon, C. A., de Kok, R., Vinatier, S., Coustenis, A., Sefton-Nash, E., Calcutt, S. B., Flasar, F. M., Nov. 2012. Active upper-atmosphere chemistry and dynamics from polar circulation reversal on Titan. Nature 491, 732-735.

Tomasko, M. G., Doose, L., Engel, S., Dafoe, L. E., West, R., Lemmon, M., Karkoschka, E., See, C., Apr. 2008. A model of Titan's aerosols based on measurements made inside the atmosphere. Planet. Space Sci. $56,669-707$.

Vinatier, S., Bézard, B., de Kok, R., Anderson, C. M., Samuelson, R. E., Nixon, C. A., Mamoutkine, A., Carlson, R. C., Jennings, D. E., Guandique, E. A., Bjoraker, G. L., Michael Flasar, F., Kunde, V. G., Dec. 2010a. Analysis of Cassini/CIRS limb spectra of Titan acquired during the nominal mission II: Aerosol extinction profiles in the $600-1420 \mathrm{~cm}^{-1}$ spectral range. Icarus $210,852-866$.

Vinatier, S., Bézard, B., Fouchet, T., Teanby, N. A., de Kok, R., Irwin, P. G. J., Conrath, B. J., Nixon, C. A., Romani, P. N., Flasar, F. M., Coustenis, A., May 2007a. Vertical abundance profiles of hydrocarbons in Titan's atmosphere at $15^{\circ} \mathrm{S}$ and $80^{\circ} \mathrm{N}$ retrieved from Cassini/CIRS spectra. Icarus 188, 120-138.

Vinatier, S., Bézard, B., Nixon, C. A., Mamoutkine, A., Carlson, R. C., Jennings, D. E., Guandique, E. A., Teanby, N. A., Bjoraker, G. L., Michael Flasar, F., Kunde, V. G., Feb. 2010b. Analysis of Cassini/CIRS limb spectra of Titan acquired during the nominal mission. I. Hydrocarbons, nitriles and $\mathrm{CO}_{2}$ vertical mixing ratio profiles. Icarus 205, 559-570.

Vinatier, S., Rannou, P., Anderson, C. M., Bézard, B., de Kok, R., Samuelson, R. E., May 2012. Optical constants of Titan's stratospheric aerosols in the $70-1500 \mathrm{~cm}^{-1}$ spectral range constrained by Cassini/CIRS observations. Icarus 219, 5-12.

Waite, J. H., Niemann, H., Yelle, R. V., Kasprzak, W. T., Cravens, T. E., Luhmann, J. G., McNutt, R. L., Ip, W.-H., Gell, D., De La Haye, V., Müller-Wordag, I., Magee, B., Borggren, N., Ledvina, S., Fletcher, G., Walter, E., Miller, R., Scherer, S., Thorpe, R., Xu, J., Block, B., Arnett, K., May 2005. Ion Neutral Mass Spectrometer Results from the First Flyby of Titan. Science 308, 982-986.

West, R. A., Balloch, J., Dumont, P., Lavvas, P., Lorenz, R., Rannou, P., Ray, T., Turtle, E. P., Mar. 2011. The evolution of Titan's detached haze layer near equinox in 2009. Geophys. Res. Let. 38, 6204.

West, R. A., Ovanessian, A., Del Genio, A., Turtle, E. P., Perry, J., NcEwen, A., Ray, T., Roy, M., Oct. 2013. Post-Equinox Evolution of Titan's Detached Haze and South Polar Vortex Cloud. In: AAS/Division for Planetary Sciences Meeting Abstracts. Vol. 45 of AAS/Division for Planetary Sciences Meeting Abstracts. p. 305.03 .

Wilson, E. H., Atreya, S. K., Jun. 2004. Current state of modeling the photochemistry of Titan's mutually dependent atmosphere and ionosphere. J. Geophys. Res. 109, doi: 10.1029/2003JE002181. E06002. 


\begin{tabular}{|c|c|c|c|c|c|c|}
\hline flyby, date & $\begin{array}{c}\text { mean } \\
\text { lat. }\end{array}$ & $\begin{array}{l}\text { mean } \\
\text { long. }\end{array}$ & $\begin{array}{l}\text { mean vertical } \\
\text { res. }(\mathrm{km})\end{array}$ & $\begin{array}{c}\text { altitude } \\
\text { range }(\mathrm{km})\end{array}$ & $\begin{array}{c}\text { number of } \\
\text { averaged spectra }\end{array}$ & $\mathbf{L}_{s}\left({ }^{\circ}\right)$ \\
\hline \multicolumn{7}{|c|}{ High northern latitudes } \\
\hline T19, $9^{\text {th }}$ Oct. 2006 & $61^{\circ} \mathrm{N}$ & $271^{\circ} \mathrm{E}$ & 38 & $143-553$ & $18-48$ & 323.5 \\
\hline T35, $30^{\text {th }}$ Aug. 2007 & $70^{\circ} \mathrm{N}$ & $102^{\circ} \mathrm{E}$ & 38 & $143-517$ & $15-53$ & 335.2 \\
\hline T59, $24^{\text {th }}$ Jul. 2009 & $67^{\circ} \mathrm{N}$ & $310^{\circ} \mathrm{E}$ & 34 & $130-517$ & $6-16$ & 359.4 \\
\hline T64, $28^{\text {th }}$ Dec. 2009 & $74^{\circ} \mathrm{N}$ & $260^{\circ} \mathrm{E}$ & 38 & $120-511$ & $29-88$ & 4.7 \\
\hline T67, $5^{\text {th }}$ Apr. 2010 & $88^{\circ} \mathrm{N}$ & $150^{\circ} \mathrm{E}$ & 38 & $141-526$ & $28-147$ & 8.1 \\
\hline T79, $13^{\text {th }}$ Dec. 2011 & $79^{\circ} \mathrm{N}$ & $99^{\circ} \mathrm{E}$ & 38 & $127-519$ & $28-134$ & 28.4 \\
\hline \multicolumn{7}{|c|}{$45^{\circ} \mathrm{N}$} \\
\hline T16, 21 th Jul. 2006 & $46^{\circ} \mathrm{N}$ & $250^{\circ} \mathrm{E}$ & 44 & $131-525$ & $23-48$ & 320.6 \\
\hline T84, $6^{\text {th }}$ Jun. 2012 & $46^{\circ} \mathrm{N}$ & $280^{\circ} \mathrm{E}$ & 38 & $120-534$ & $33-72$ & 34.1 \\
\hline \multicolumn{7}{|c|}{ Equator } \\
\hline T23, $13^{\text {th }}$ Jan. 2007 & $4^{\circ} \mathrm{N}$ & $33^{\circ} \mathrm{E}$ & 38 & $148-531$ & $21-80$ & 327.0 \\
\hline T83, $21^{\text {th }}$ May 2012 & $0^{\circ} \mathrm{N}$ & $106^{\circ} \mathrm{E}$ & 36 & $143-550$ & $17-123$ & 33.6 \\
\hline & & & $45^{\circ} \mathrm{S}$ & & & \\
\hline T39, $21^{\text {th }}$ Dec. 2007 & $46^{\circ} \mathrm{S}$ & $288^{\circ} \mathrm{E}$ & 44 & $98-517$ & $29-84$ & 339.2 \\
\hline T82, $18^{\text {th }}$ Feb. 2012 & $46^{\circ} \mathrm{S}$ & $57^{\circ} \mathrm{E}$ & 38 & $111-530$ & $29-103$ & 30.6 \\
\hline \multicolumn{7}{|c|}{ High southern latitudes } \\
\hline T65, $12^{\text {th }}$ Jan. 2010 & $76^{\circ} \mathrm{S}$ & $60^{\circ} \mathrm{E}$ & 37 & $118-500$ & $29-85$ & 5.3 \\
\hline T78, $11^{\text {th }}$ Sep. 2011 & $84^{\circ} \mathrm{S}$ & $220^{\circ} \mathrm{E}$ & 36 & $109-528$ & $27-105$ & 25.4 \\
\hline
\end{tabular}

Table 1: Characteristics of the averaged limb spectra acquired at a spectral resolution of $0.5 \mathrm{~cm}^{-1}$. The first four columns give the Titan flyby number and its date, the mean latitude, longitude and vertical resolution of the limb spectra. The fifth column gives the minimum and maximum altitudes of the line-of-sight of the averaged spectra. Sixth column gives the minimum and maximum number per averaged spectra. Last column gives the planetocentric orbital longitude of the Sun. Spectra were extracted from the 3.2 version of the CIRS database. 


\begin{tabular}{|c|c|c|c|c|}
\hline flyby, date & $\begin{array}{l}\text { latitudes } \\
\left({ }^{\circ} \mathrm{N}\right)\end{array}$ & $\begin{array}{l}\text { longitudes } \\
\left({ }^{\circ} \mathrm{E}\right)\end{array}$ & $\begin{array}{l}\text { vertical } \\
\text { solution }(\mathrm{km})\end{array}$ & $\mathbf{L}_{s}\left({ }^{\circ}\right)$ \\
\hline T59, 2009 July 24 & $0,15,31,41,51,61$ & $225,232,241,250,260,280$ & $48,45,40,37,35,32$ & 359.4 \\
\hline T63, 2009 Dec. 11 & $\begin{array}{c}4,15,25,35,45 \\
55,65,75,85\end{array}$ & $\begin{array}{c}107,108,108,108,108 \\
107,107,106,106\end{array}$ & $\begin{array}{c}31,33,35,37,39 \\
42,44,46,48\end{array}$ & 4.2 \\
\hline T65, 2010 Jan. 13 & $\begin{array}{l}-85,-80,-75,-65,-55 \\
-45,-35,-25,-15,-5\end{array}$ & $\begin{array}{c}340,320,315,309,307 \\
305,304,303,303,303\end{array}$ & $\begin{array}{l}28,29,30,33,35 \\
36,39,41,44,46\end{array}$ & 5.3 \\
\hline T69, 2010 June 4 & $\begin{array}{l}-85,-80,-75,-70,-60,-50 \\
-40,-30,-20,-10,-2\end{array}$ & $\begin{array}{c}160,130,123,118,115,113 \\
111,110,110,110,110\end{array}$ & $\begin{array}{c}50,49,48,46,44,42 \\
39,37,34,32,31\end{array}$ & 10.1 \\
\hline T70, 2010 June 20 & $\begin{array}{r}5,15,35,40,50 \\
60,70,75,80,85\end{array}$ & $\begin{array}{l}290,288,288,288,288 \\
288,287,286,285,280\end{array}$ & $\begin{array}{l}47,45,40,39,36 \\
34,32,31,30,28\end{array}$ & 10.6 \\
\hline T76, 2011 May 8 & $\begin{array}{l}10,20,30,40,50 \\
60,65,75,80,85\end{array}$ & $\begin{array}{l}60,60,60,60,61 \\
62,63,65,68,75\end{array}$ & $\begin{array}{l}46,45,42,40,37 \\
35,34,32,31,29\end{array}$ & 21.3 \\
\hline T77, 2011 June 20 & $\begin{array}{c}-85,-80,-75,-70,-65,-60,-55 \\
-45,-35,-30,-20,-10,0\end{array}$ & $\begin{array}{c}127,125,125,119,118,118,117 \\
116,116,115,115,115,114\end{array}$ & $\begin{array}{c}47,46,45,44,43,41,40 \\
40,36,35,33,30,28\end{array}$ & 22.7 \\
\hline T80, 2012 Jan. 2 & $\begin{array}{c}0,10,20,25,35 \\
45,55,60,65,75\end{array}$ & $\begin{array}{c}68,69,71,72,74 \\
76,80,83,87,105\end{array}$ & $\begin{array}{l}32,34,36,37,40 \\
42,44,45,46,48\end{array}$ & 29.0 \\
\hline T82, 2012 Feb. 19 & $\begin{array}{l}-80,-75,-70,-65,-60,-55 \\
-50,-40,-30,-20,-15,-5\end{array}$ & $\begin{array}{l}256,254,253,252,252,252 \\
251,250,249,249,245,245\end{array}$ & $\begin{array}{l}47,46,45,44,43,41 \\
40,38,36,33,32,30\end{array}$ & 30.6 \\
\hline T85, 2012 July & $\begin{array}{c}-15,-10,0,10,20 \\
30,40,50,60,65\end{array}$ & $\begin{array}{l}111,113,117,122,127 \\
130,138,147,160,180\end{array}$ & $\begin{array}{l}28,29,31,33,36 \\
38,40,42,45,46\end{array}$ & 35.6 \\
\hline T88, 2012 Nov. & $\begin{array}{c}-50,-45,-40,-35,-30,-20 \\
\quad-10,0,10,20,30\end{array}$ & $\begin{array}{c}355,340,332,327,320,315 \\
305,300,295,285,278 \\
\end{array}$ & $\begin{array}{c}47,46,45,43,42,40 \\
37,36,34,31,29 \\
\end{array}$ & 39.6 \\
\hline T91, 2013 May 23 & $-25,-20,-15,0,10,20$ & $350,340,337,325,315,305$ & $35,36,37,40,43,46$ & 45.2 \\
\hline
\end{tabular}

Table 2: Characteristics of the averaged limb spectra acquired at a spectral resolution of $15.5 \mathrm{~cm}^{-1}$. First column gives the Titan flyby number and its date, second and third columns gives respectively the latitude and longitudes of each limb observation used to infer global maps of temperature, aerosol extinction and molecular mixing ratio. Fourth column gives the vertical resolution of the corresponding limb observations. Last column gives the planetocentric orbital longitude of the Sun at the date of the flyby. Spectra were extracted from the 3.2 version of the CIRS database. 

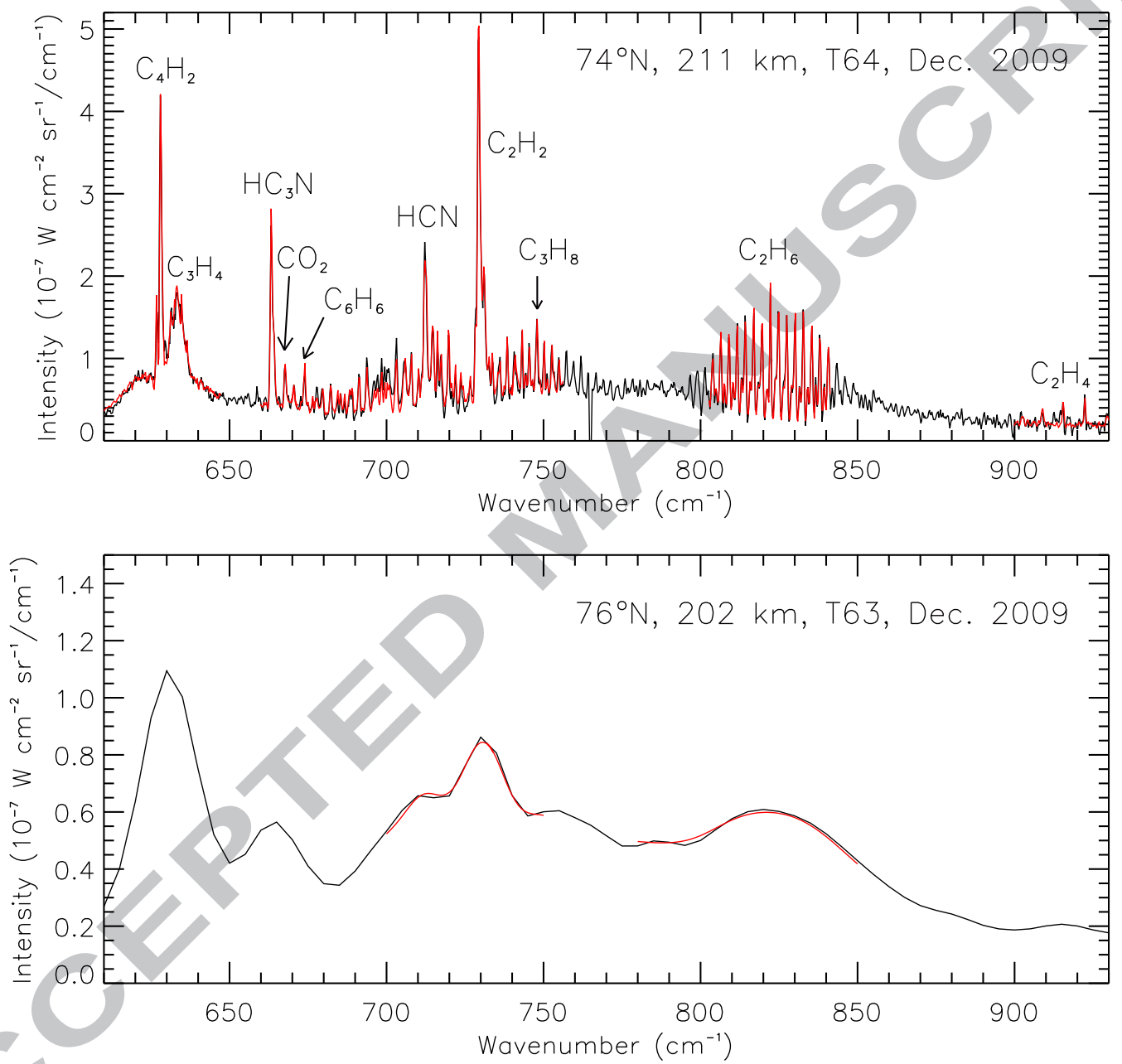

Figure 1: Upper panel: limb spectrum acquired at $211 \mathrm{~km}$ at $74^{\circ} \mathrm{N}$ in December 2009, during the T64 flyby. The spectral resolution is $0.5 \mathrm{~cm}^{-1}$. The calculated spectrum, unsing the mixing ratio profiles displayed in Fig. 10 are shown in red. Lower panel: limb spectrum acquired at $202 \mathrm{~km}$ at $76^{\circ} \mathrm{N}$ in December 2009 , during the T63 flyby. The spectral resolution is $15.5 \mathrm{~cm}^{-1}$. The calculated spectrum, using the mixing ratio profiles displayed in the map of Figs 3,5 and 4 are shown in red. Note that the y-scale differs from that of the upper panel. 

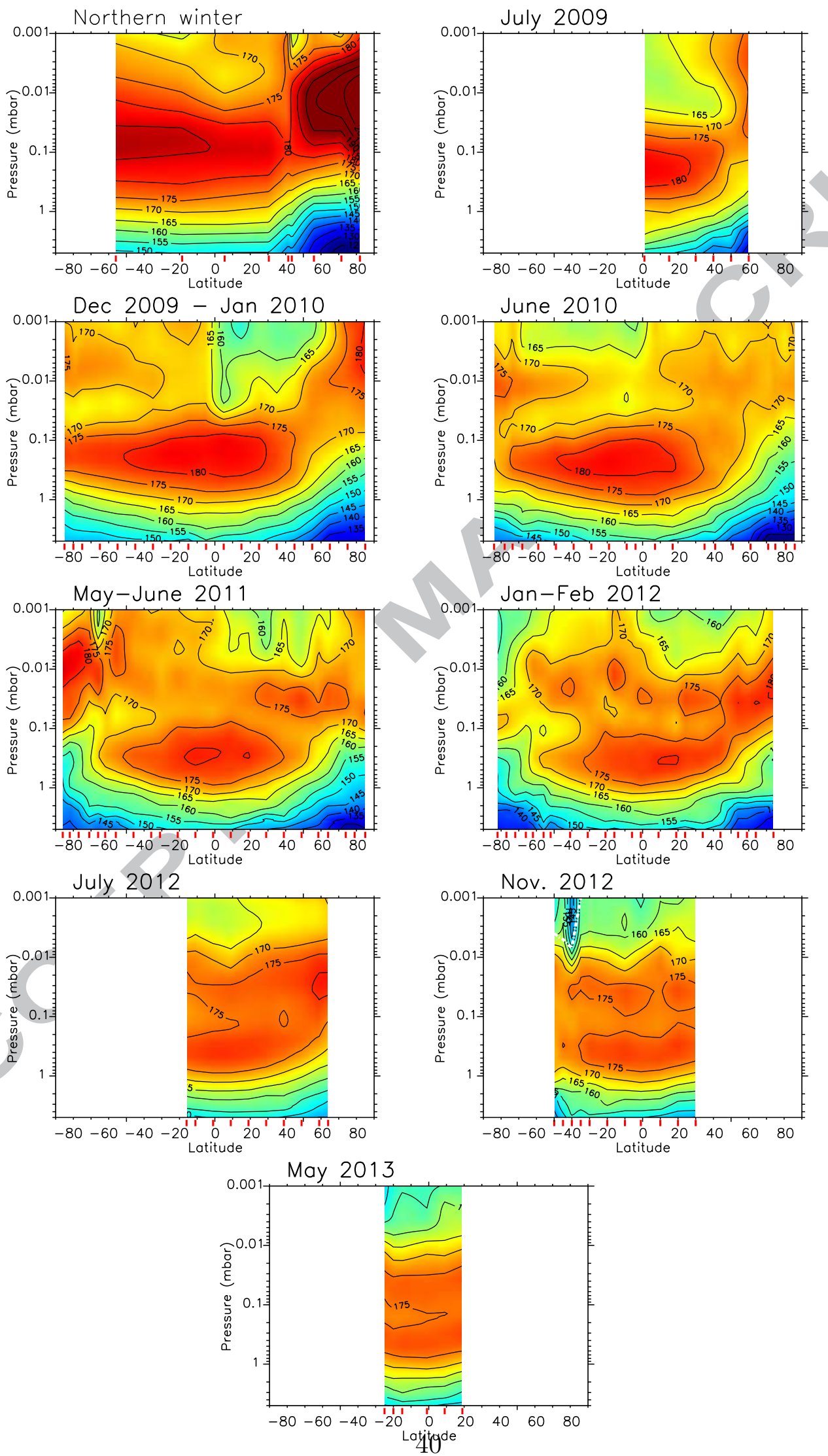

Figure 2: Pressure/latitudes maps of temperature. Red marks on the horizontal scale give the latitudes where limb spectra were analyzed. White areas indicate regions were CIRS did not acquire limb spectra. White dashed lines show the levels above which the constraints on the thermal profiles are poor (typically because of a too low signal-to-noise ratio). 

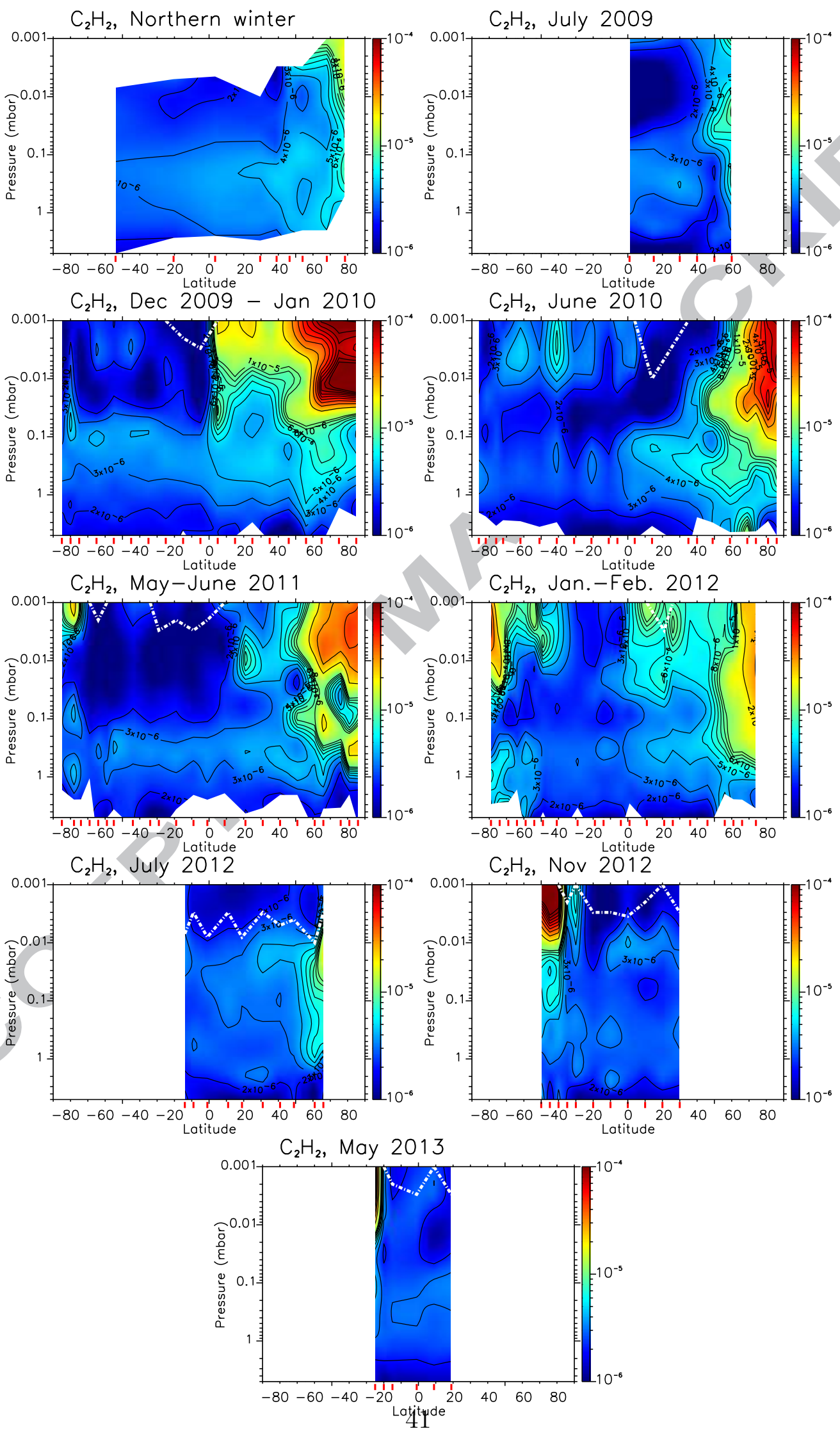

Figure 3: Pressure/latitudes maps of the $\mathrm{C}_{2} \mathrm{H}_{2}$ mixing ratio. Red marks on the horizontal scale give the latitudes where limb spectra were analyzed. White areas indicate regions were CIRS did not acquire limb spectra or were the extinction profile is not constrained from the data. White dashed lines show the levels above which the constrains on the mixing ratio are poor (typically because of a too low signal-to-noise ratio). 

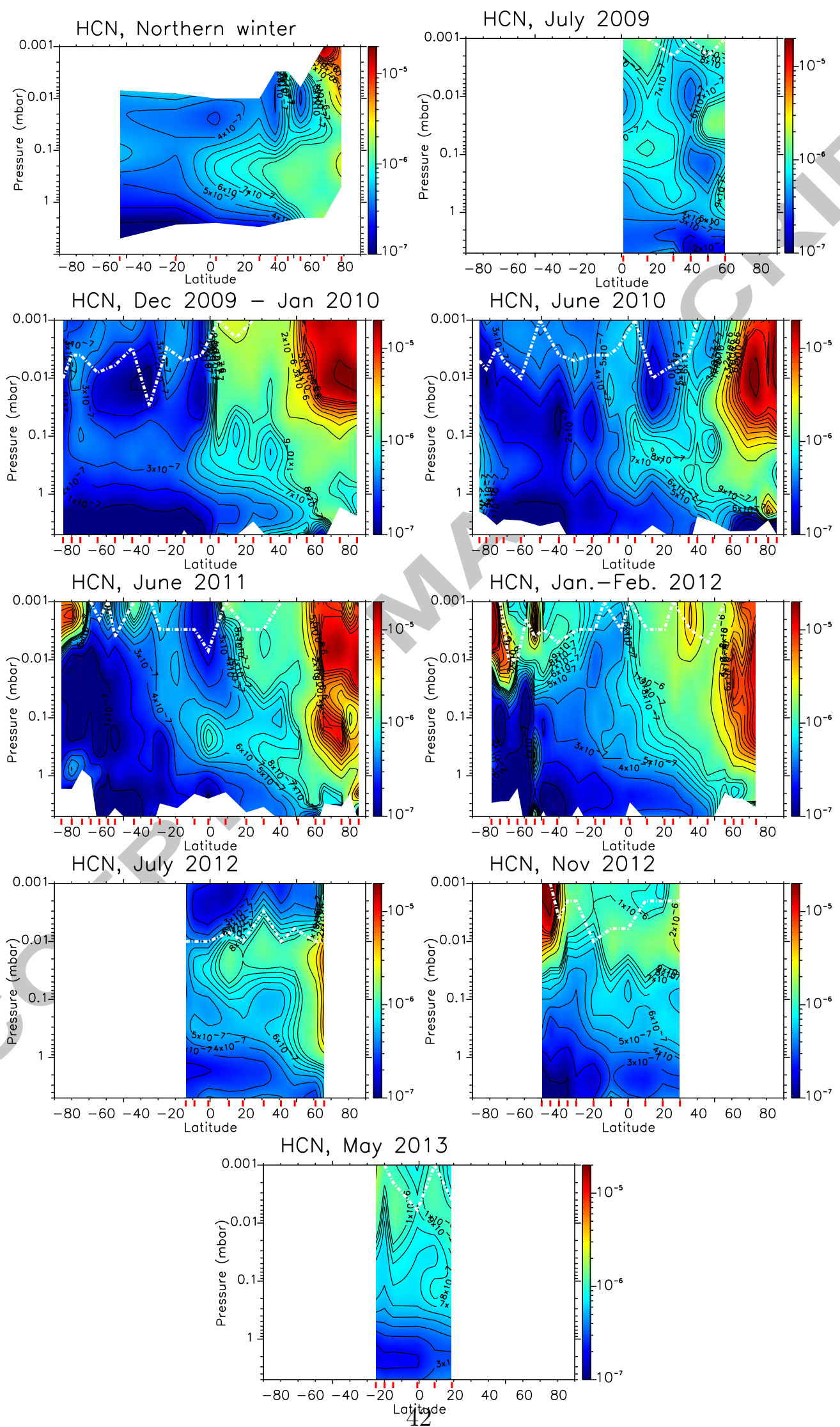

Figure 4: Similar to Fig. 3 but for HCN. 

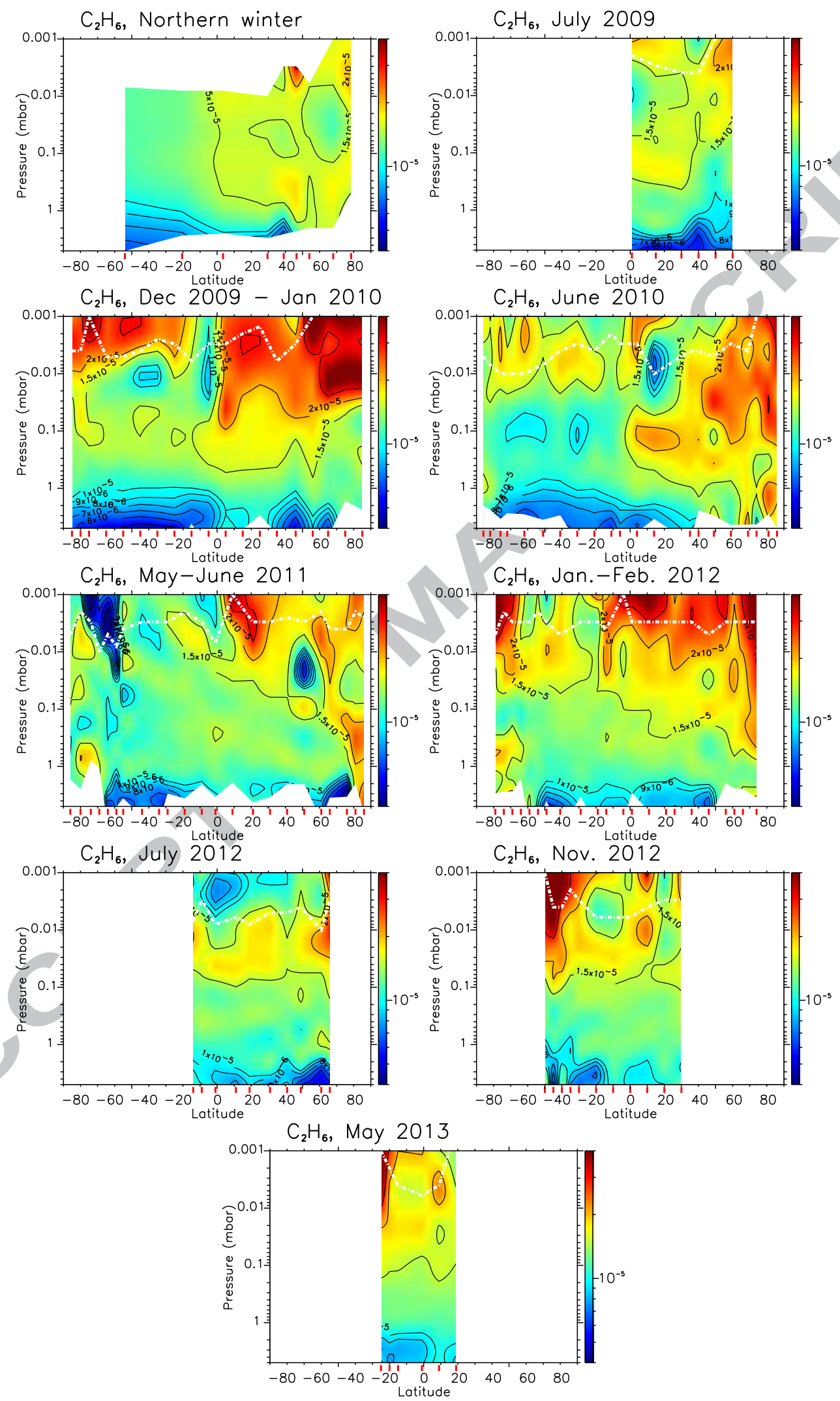

Figure 5: Similar to4ङ3ig. 3 but for $\mathrm{C}_{2} \mathrm{H}_{6}$. 

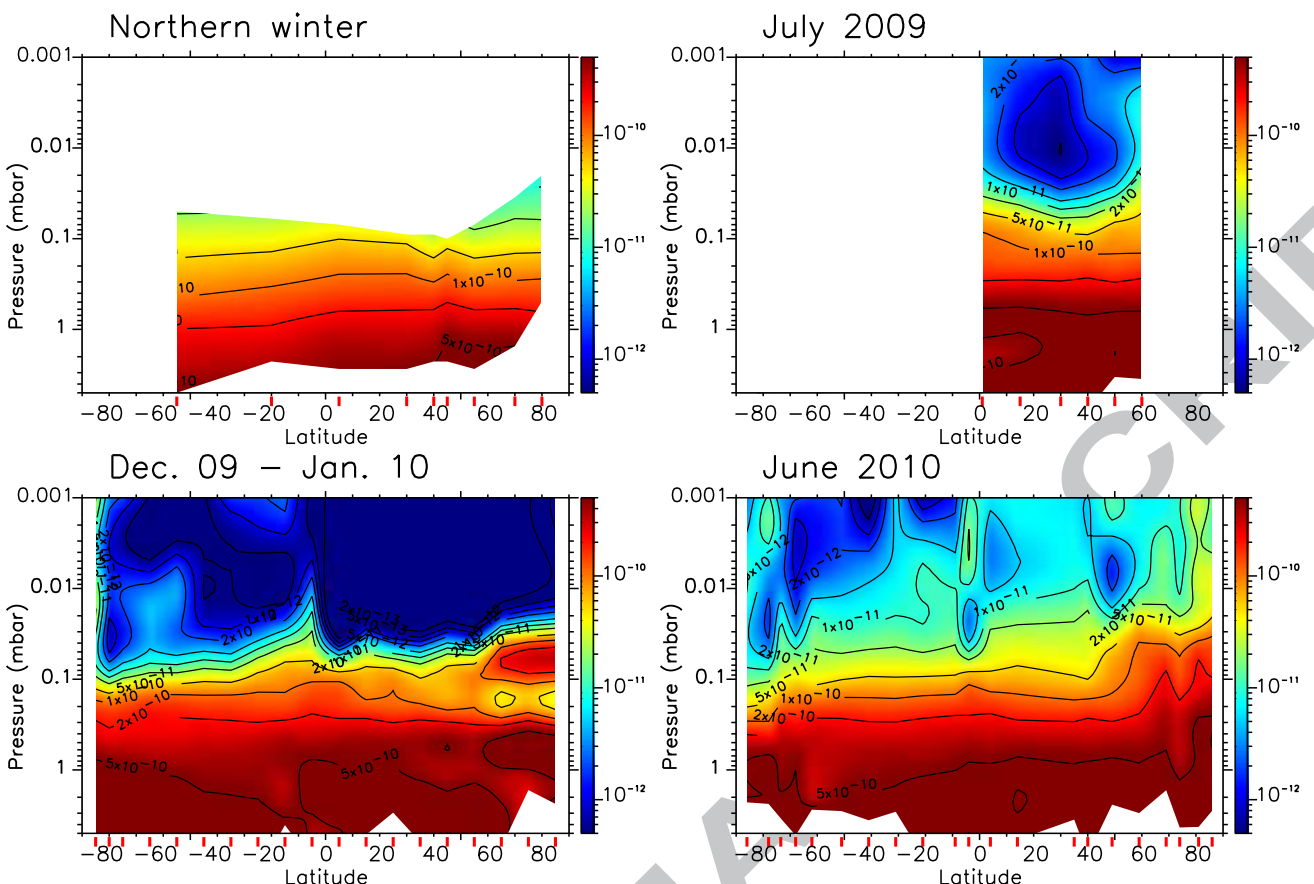

June 2010
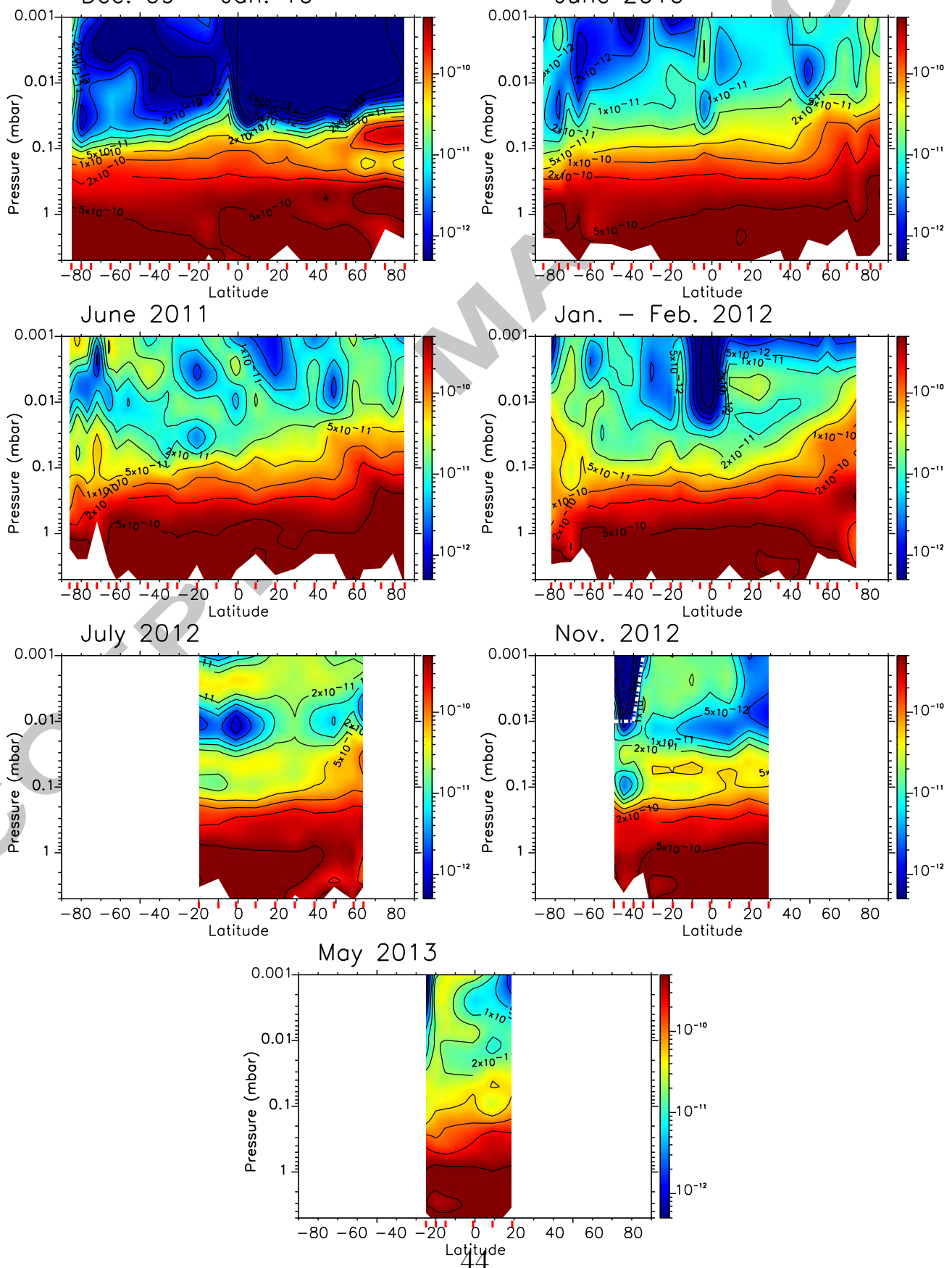

Figure 6: Pressure/latitudes maps of the haze extinction coefficient. Values are given in $\mathrm{cm}^{-1}$ for the 1090 $\mathrm{cm}^{-1}$ wavenumber. Red marks on the horizontal scale give the latitudes where limb spectra were analyzed. White areas indicate regions were CIRS did not acquire limb spectra or were the extinction profile is not constrained from the data. White dashed lines show the levels above which the constraints on the extinction coefficient are poor (typically because of a too low signal-to-noise ratio). 

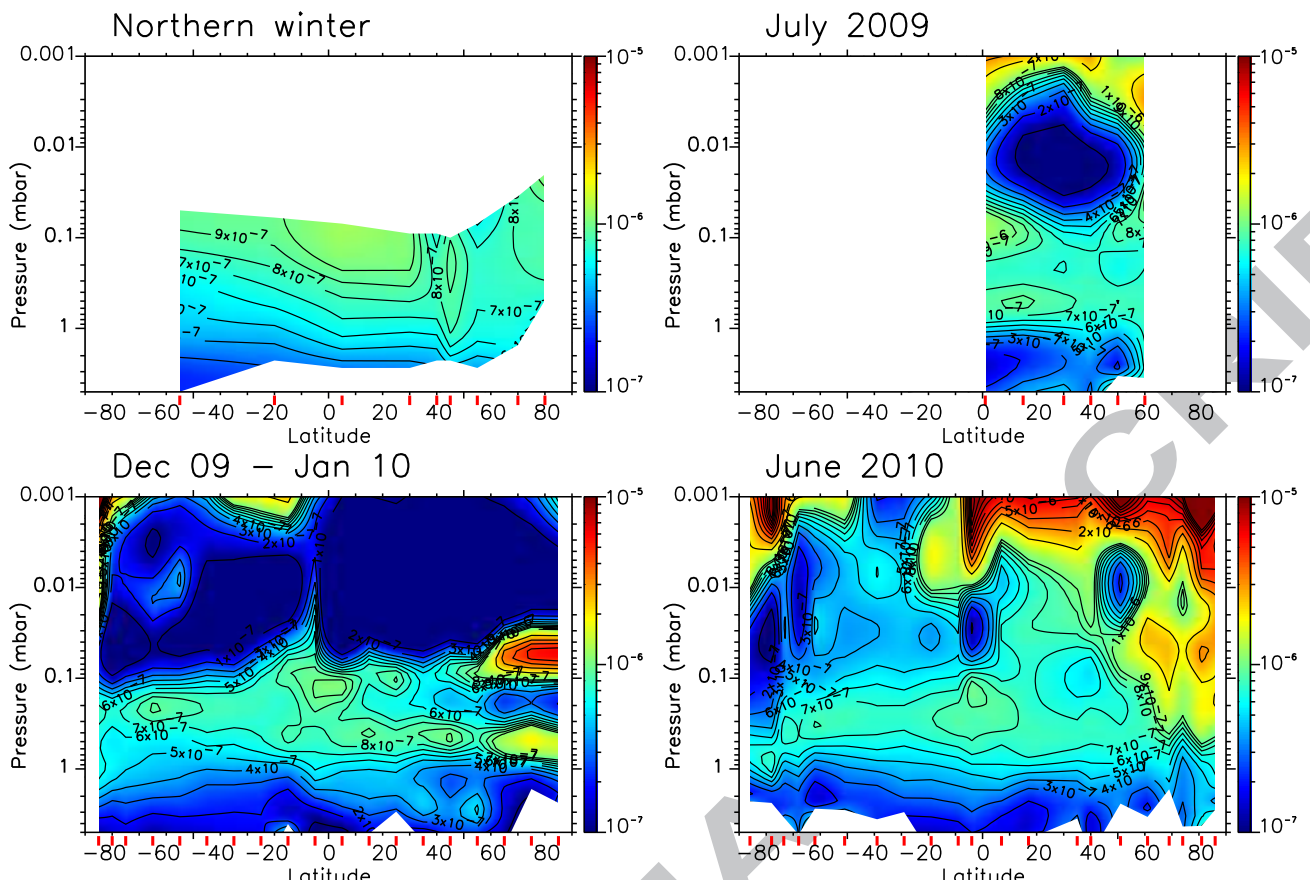

June 2010

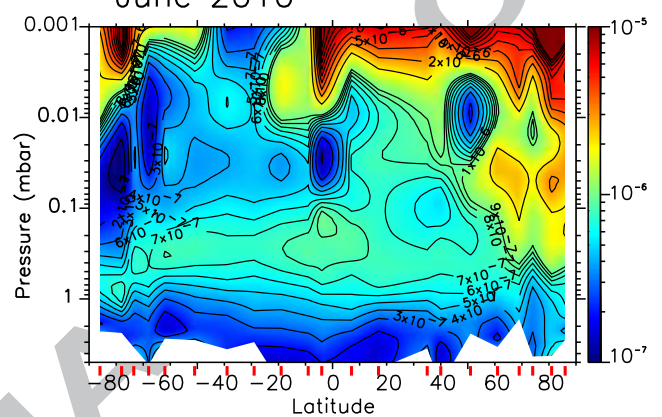

June. 2011

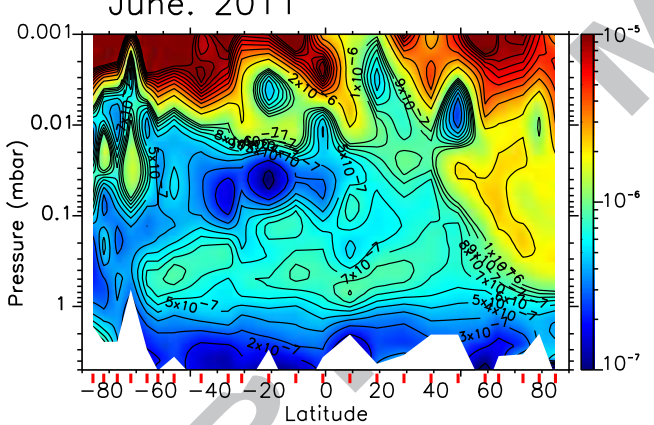

Jan. - Feb. 2012
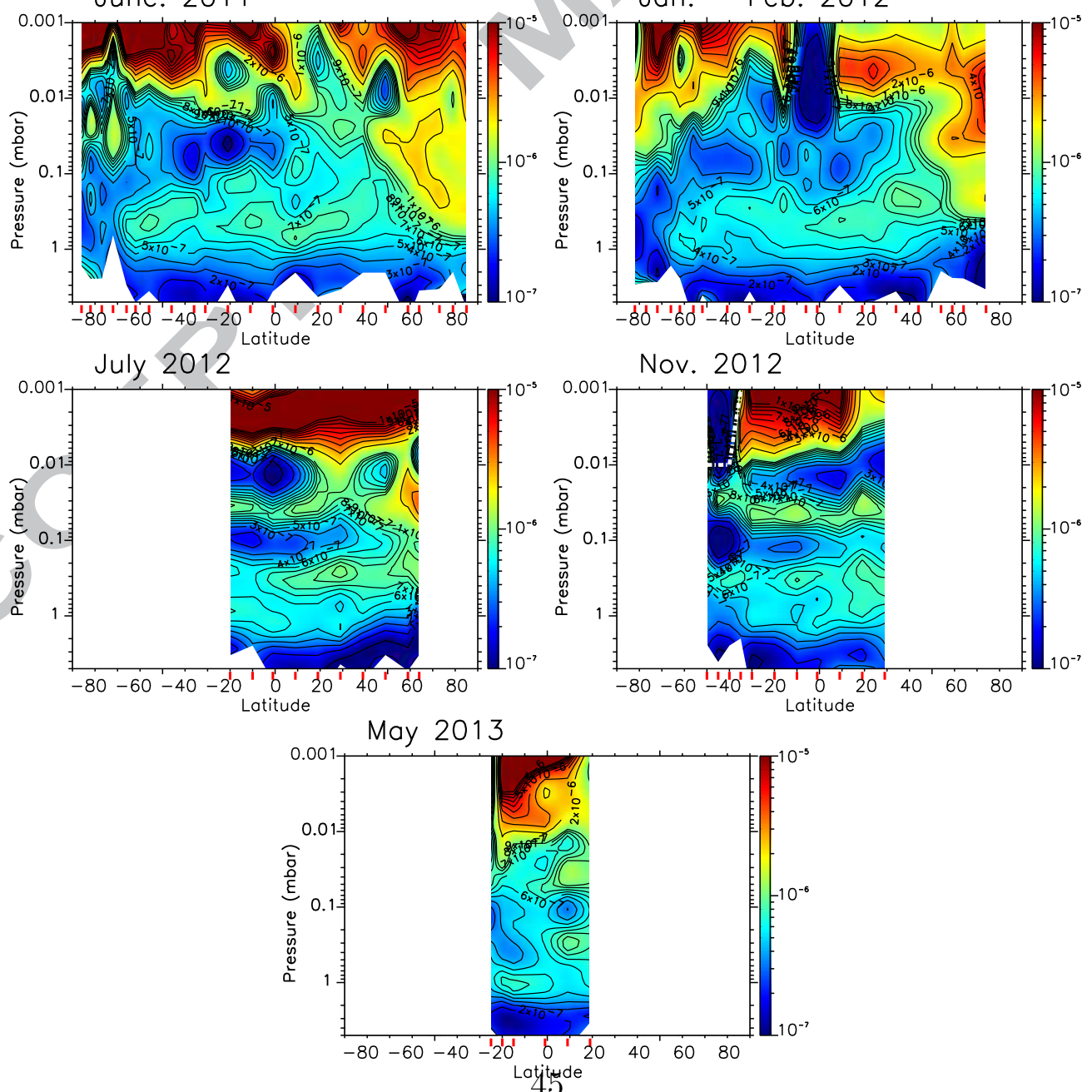

Figure 7: Pressure/latitudes maps of the haze mass mixing ratio. Red marks on the horizontal scale give the latitudes where limb spectra were analyzed. White areas indicate regions where CIRS did not acquire limb spectra or where the extinction profile is not constrained from the data. White dashed lines show the levels above which the constraints on the aerosol mass mixing ratio are poor (typically because of a too low signal-to-noise ratio). 


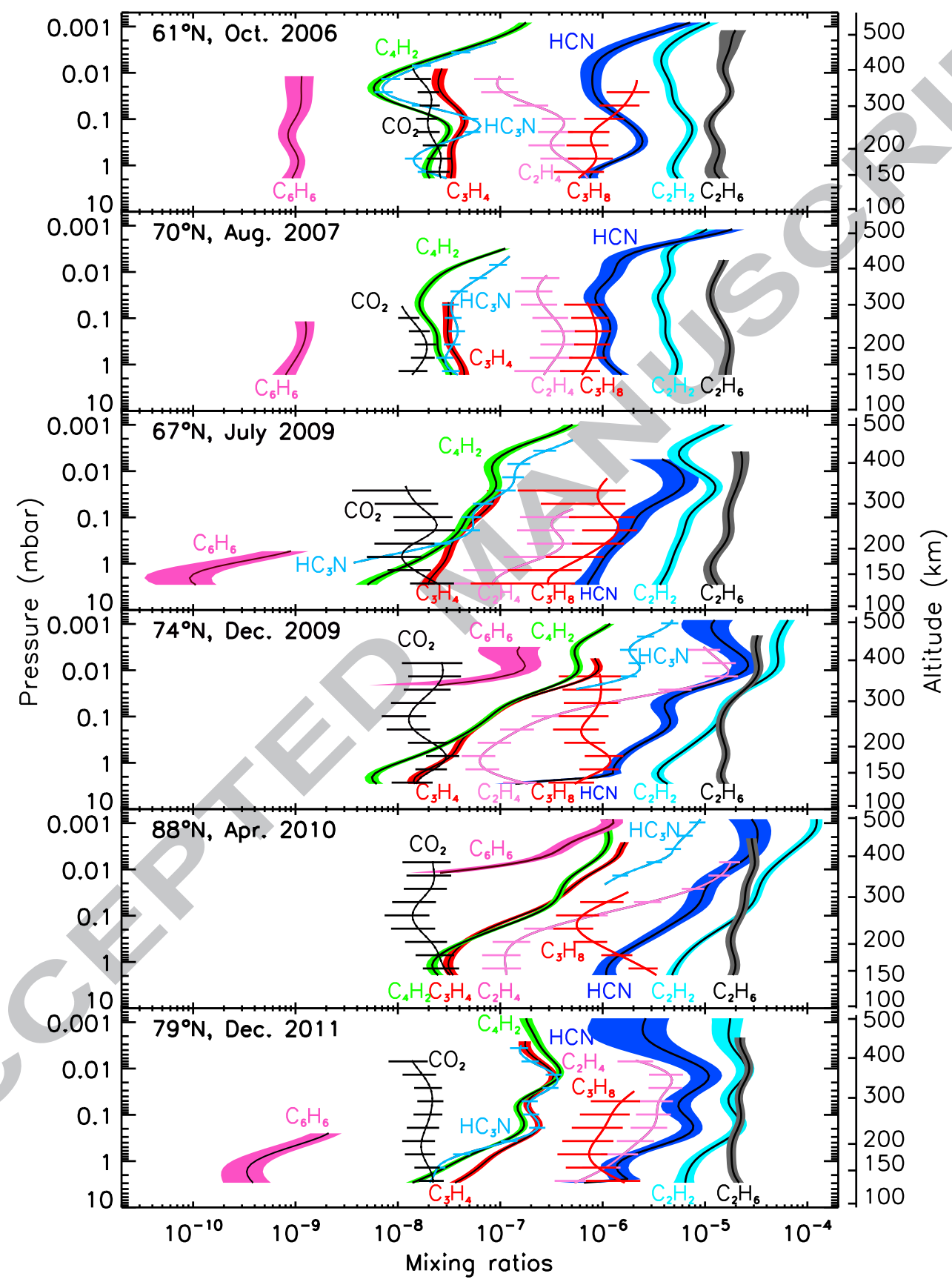

Figure 8: Seasonal variations of the mixing ratio profiles at the north pole, derived from $0.5 \mathrm{~cm}^{-1}$ resolution limb spectra. Envelops and horizontal bars give the $1-\sigma$ error bars. 


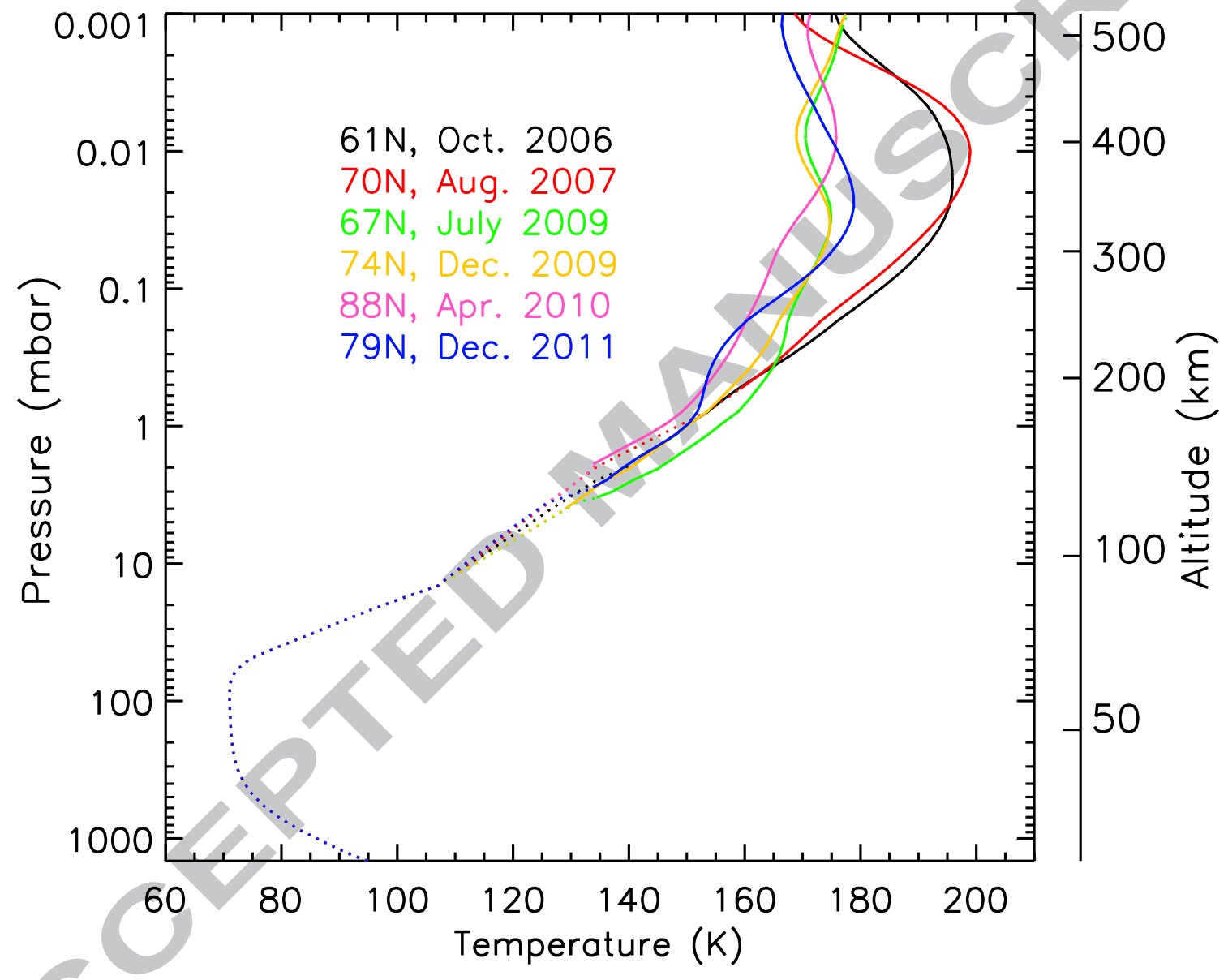

Figure 9: Retrieved temperature profiles from the $0.5 \mathrm{~cm}^{-1}$ resolution limb spectra (solid lines) at high northern latitudes during the northern winter (black and red), just before (green) and just after (yellow) the northern spring equinox and later in the spring are plotted (pink and blue). 1- $\sigma$ error bars on the temperature are lower than $1 \mathrm{~K}$. Dotted lines show region where there are no constraints from CIRS limb spectra. 

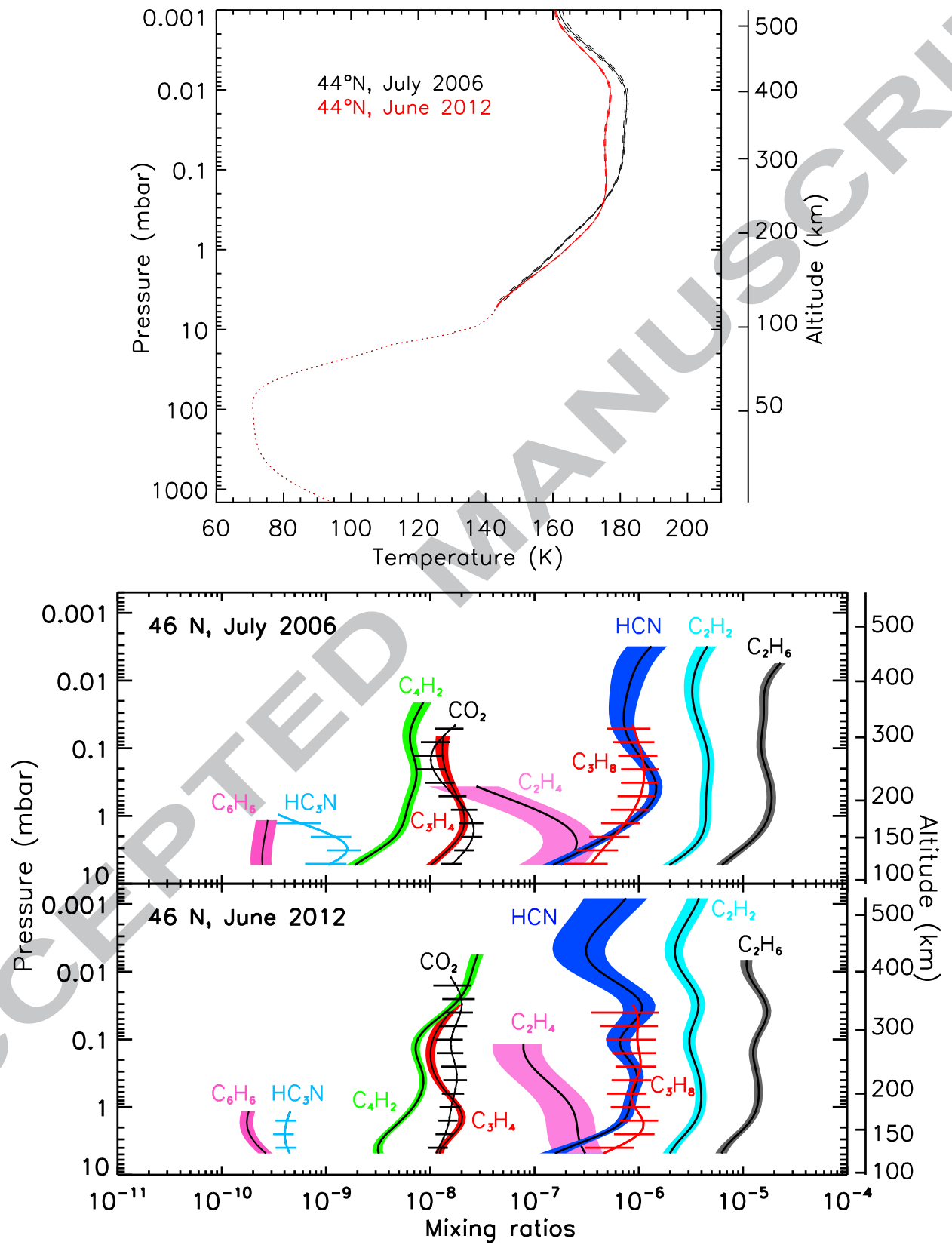

Figure 10: Seasonal variations of the thermal and mixing ratio profiles at $45^{\circ} \mathrm{N}$, derived from $0.5 \mathrm{~cm}^{-1}$ resolution limb spectra. 1- $\sigma$ error bars are given as dashed lines for the thermal profiles and envelopes or horizontal bars for the mixing ratio profiles. 

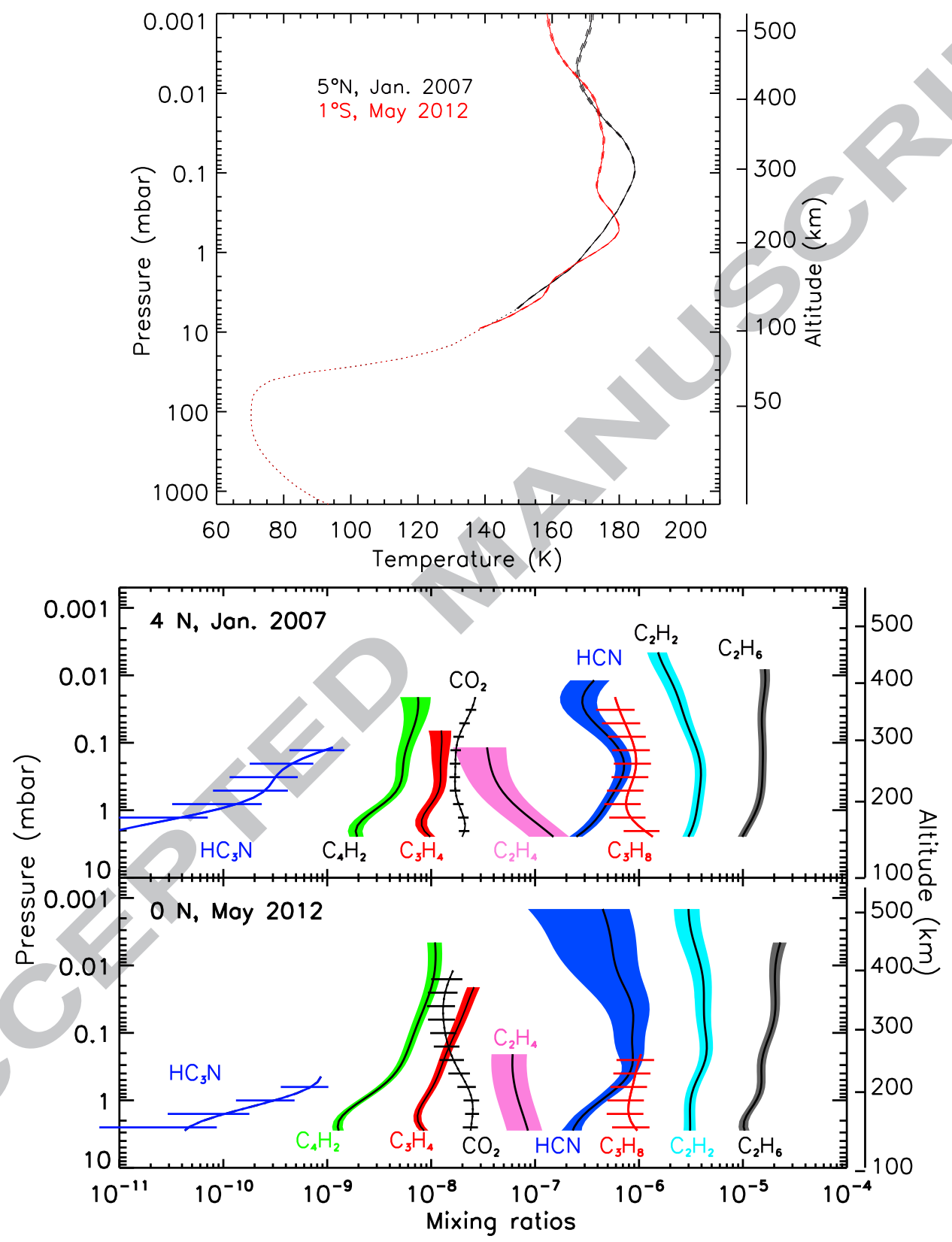

Figure 11: Seasonal variations of the thermal and mixing ratio profiles at the equator, derived from 0.5 $\mathrm{cm}^{-1}$ resolution limb spectra. 1- $\sigma$ error bars are given as dashed lines for the thermal profiles and envelopes or horizontal bars for the mixing ratio profiles. 

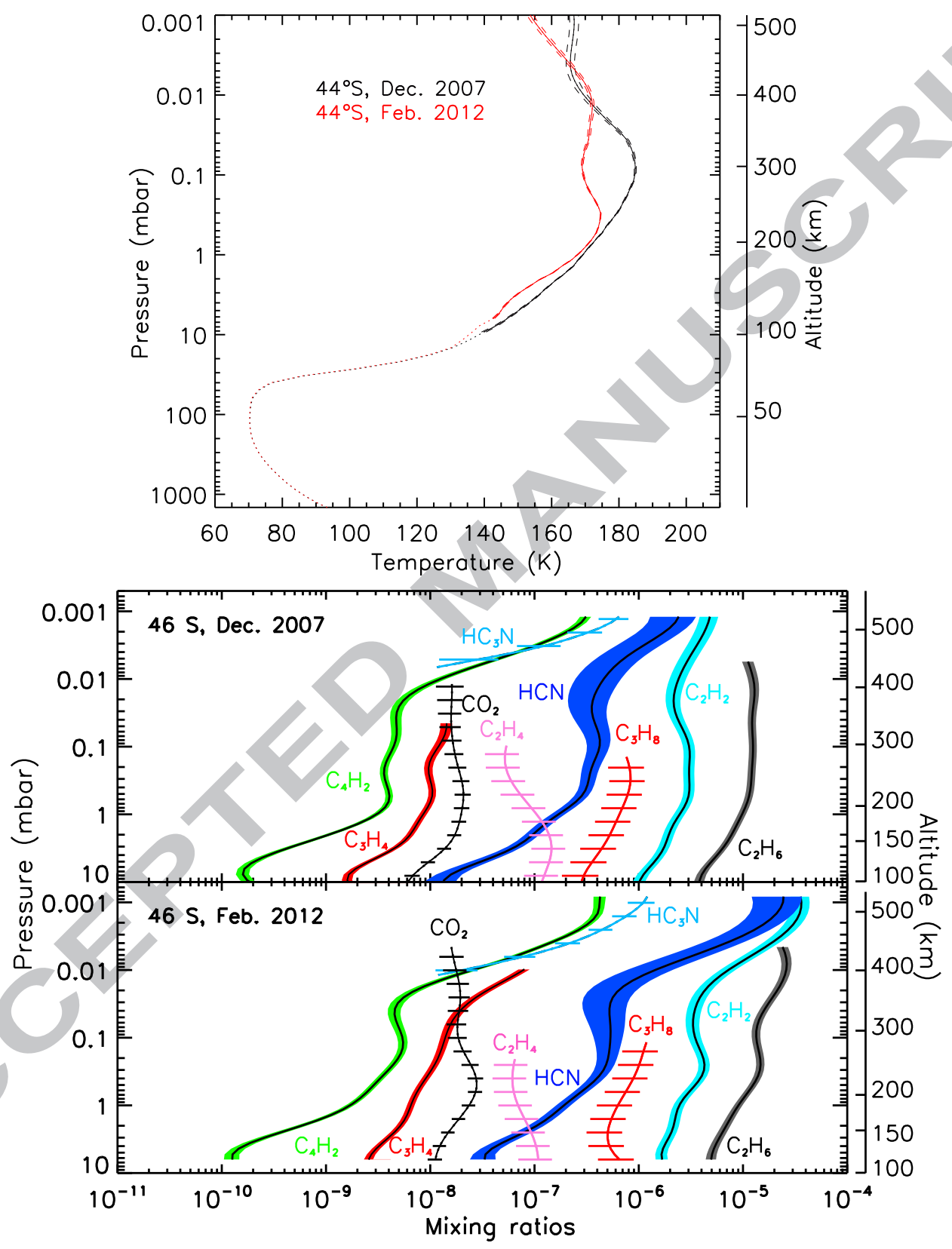

Figure 12: Seasonal variations of the thermal and mixing ratio profiles at $45^{\circ} \mathrm{S}$, derived from $0.5 \mathrm{~cm}^{-1}$ resolution limb spectra. 1- $\sigma$ error bars are given as dashed lines for the thermal profiles and envelopes or horizontal bars for the mixing ratio profiles. 

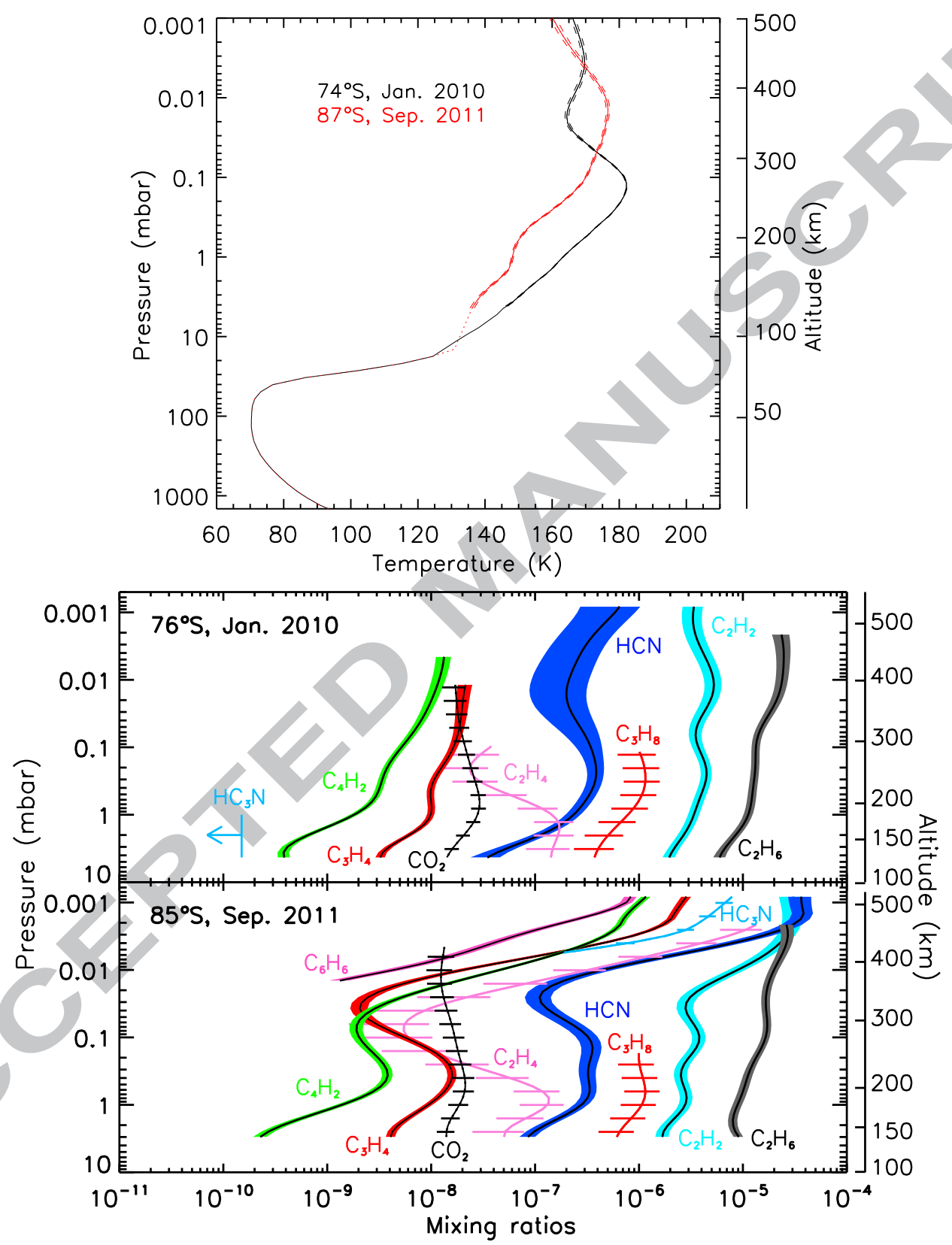

Figure 13: Seasonal variations of the thermal and mixing ratio profiles at high southern latitude, derived from $0.5 \mathrm{~cm}^{-1}$ resolution limb spectra. 1- $\sigma$ error bars are given as dashed lines for the thermal profiles and envelopes or horizontal bars for the mixing ratio profiles. 

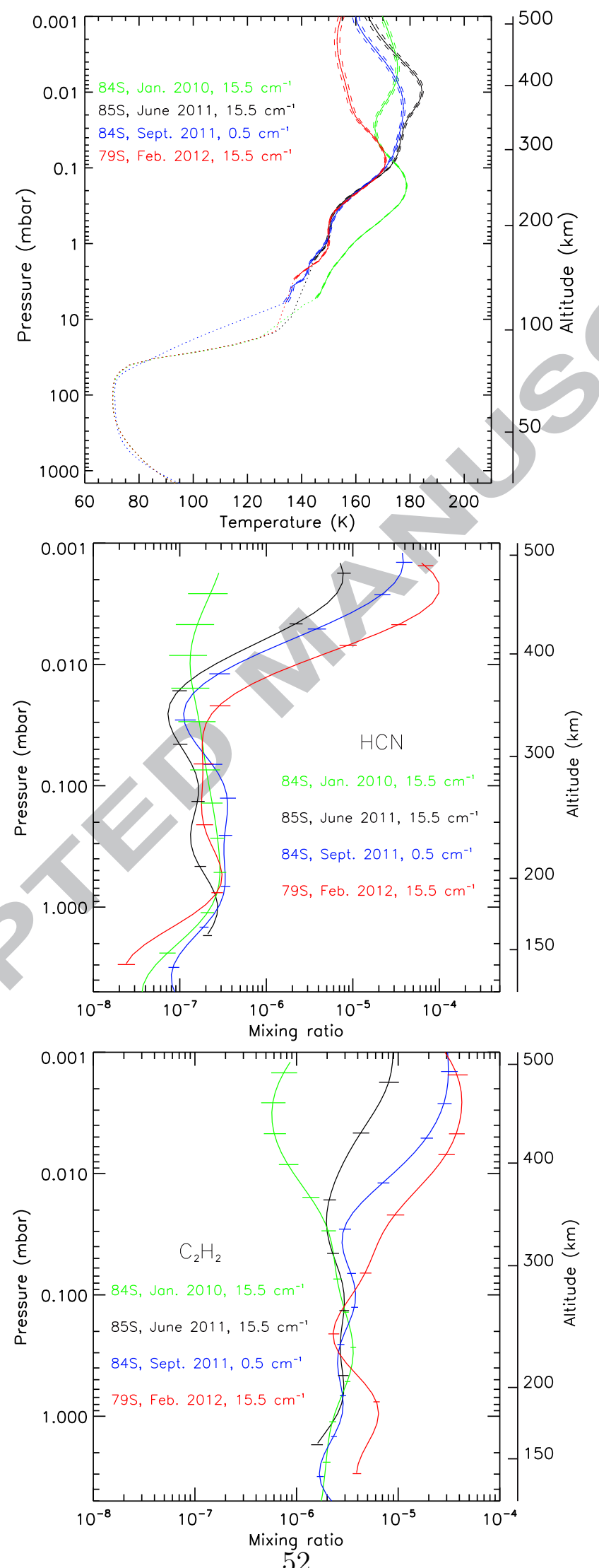

Figure 14: Seasonal evolution of the temperature profiles and the mixing ratio profiles of $\mathrm{HCN}$ and $\mathrm{C}_{2} \mathrm{H}_{2}$ at very high southern latitudes between January 2010 and February 2012. Dashed lines give the 1- $\sigma$ uncertainty on the thermal profile. For $\mathrm{HCN}$ and $\mathrm{C}_{2} \mathrm{H}_{2}$ mixing ratios, the 1- $\sigma$ error bars are plotted at the altitude of the line-of-sight of each limb spectra used for each profile retrieval. 

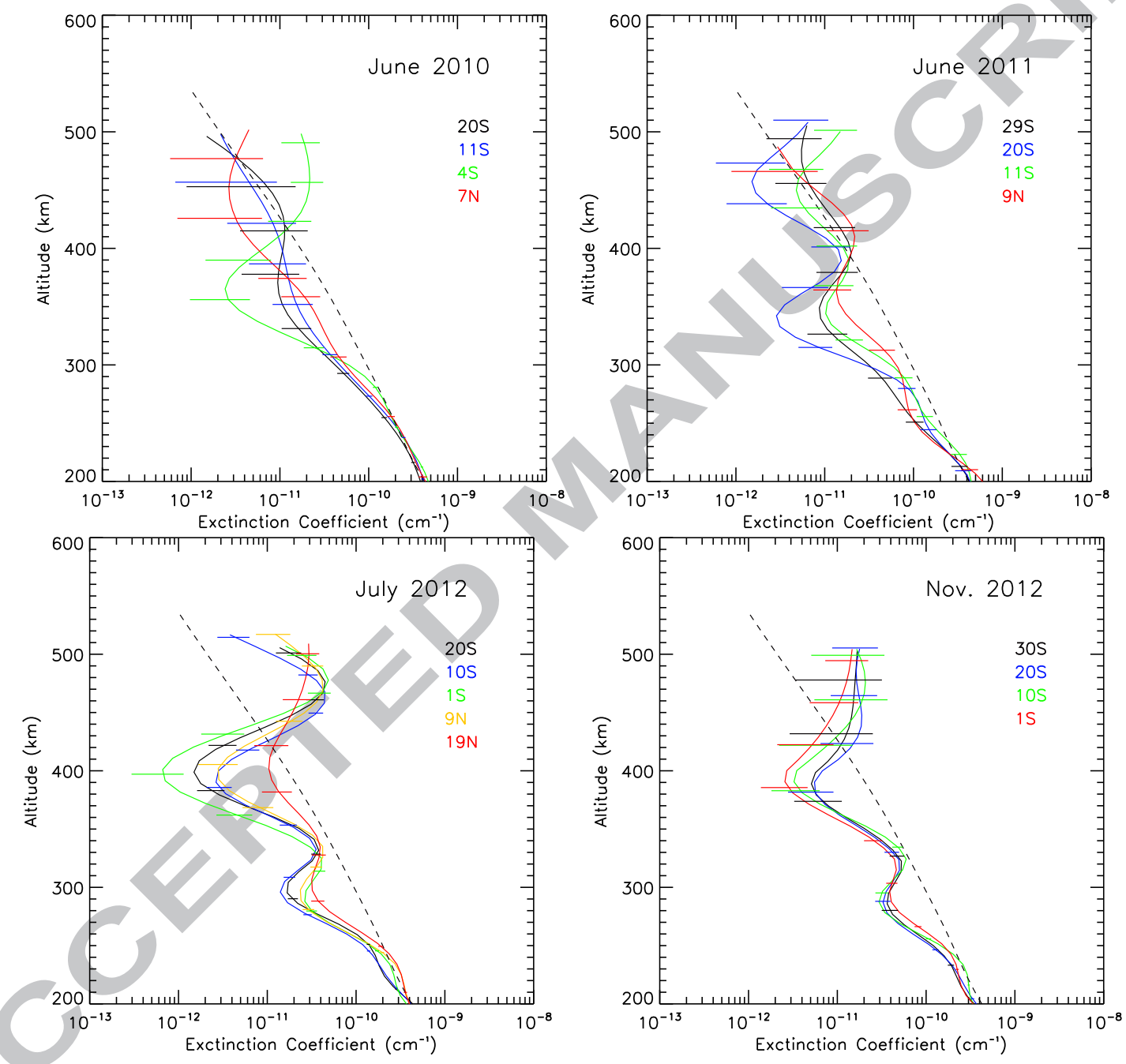

Figure 15: Aerosol extinction profiles at $1090 \mathrm{~cm}^{-1}$ in June 2010, June 2011, July 2012 and November 2012 in the equatorial region. For comparison, the extinction profile at $5^{\circ} \mathrm{N}$ in 2007 adapted from Vinatier et al. (2010a), is plotted as black dashed line. Error bars, which give the 1- $\sigma$ uncertainty, are plotted at the altitude of the line-of-sight of each limb spectra used for each profile retrieval. 
Two-cell dynamics exists in Titan's stratosphere from Jan. 2010 to June 2011.

Reversal of the global dynamics occurs within 2 years after northern spring equinox.

Timing of the dynamics changes agrees with General Circulation Model predictions.

An unexpected cooling, starting in 2012, is observed at the south pole above $350 \mathrm{~km}$. 